Bangladesh J. Plant Taxon. 27(1): 153-171, 2020 (June)

(C) 2020 Bangladesh Association of Plant Taxonomists

\title{
ANGIOSPERMS IN NARSINGDI DISTRICT OF BANGLADESH: CLASS MAGNOLIOPSIDA
}

\author{
Robayda Khanam*, Saleh Ahammad Khan and Md. Abdur Rahim \\ Plant Systematics and Biodiversity Laboratory, Department of Botany, \\ Jahangirnagar University, Savar, Dhaka, Bangladesh
}

Keywords: Angiosperms; Magnoliopsida; Narsingdi; Bangladesh.

\begin{abstract}
This study presents taxonomic information on 468 plant species belonging to 326 genera and 85 families of the angiospermic class Magnoliopsida (dicotyledones) from Narsingdi district of Bangladesh. In this area, Fabaceae with 53 species of 37 genera is the largest family, followed by Asteraceae with 25 species of 24 genera and Rubiaceae with 21 species of 17 genera. Ficus with eight species is appeared as the largest genus, which is followed by Persicaria and Solanum with seven species each, Lindernia with six species, Phyllanthus with five species, and Acacia, Amaranthus, Senna, Sida and Trichosanthes with four species each. Herbs are comprised of $230(49.14 \%)$ species that are followed by trees of $120(25.64 \%)$ species, and shrubs of $118(25.21 \%)$ species. The similarity measured by Jaccard coefficient shows that the six upazilas of Narsingdi district are relatively more similar $(75.47 \%)$ in their species composition if they are compared all together. However, the similarities vary from $9.03 \%$ to $50 \%$ if only two upazilas are compared. In this district, total 333 species have been distinguished as economically useful. The species Rauvolfia serpentina of Apocynaceae, Geophila repens of Rubiaceae and Acmella radicans of Asteraceae have been assessed as Endangered (E) in this district.
\end{abstract}

\section{Introduction}

Bangladesh constitutes a significant part of the South Asian Mega Centre of genetic diversity (Chowdhury, 1996), and supports a rich biodiversity including a huge number (approx. 5000) of Angiosperm species (Khan, 1977). However, Angiosperms of many areas of this country have so far been poorly or partially explored or unexplored. Therefore, performing taxonomic studies in these areas is essential to know the status of plant resources of this country.

Narsingdi is one of the central districts of Bangladesh, located at $50 \mathrm{~km}$ northeast of the capital city Dhaka. Some of the previous floristic studies cover the area of the present political boundary of Bangladesh (Prain, 1903; Hooker, 1872-1897; Ahmed et al., 2008-2009). Many other studies were conducted in the different areas of this country (Rahman and Hassan, 1995; Rashid, et al., 1995; Rashid and Mia, 2001; Khan and Huq 2001; Alam et al. 2006; Islam et al. 2009; Tutul et al. 2010; Arefin et al. 2011; Rahman et al. 2012; Rahman, 2013; Sarker et al. 2013; Rahman et al. 2015; Haque et al., 2016, 2018; Shetu et al. 2018). However, the flora and plant diversity of Narsingdi district have not yet been studied based on field inventories and examination of plant specimens. Moreover, this district is also one of the most risk prone areas of Bangladesh because most of its areas harboring the natural vegetation are continuousely being replaced by urbanization with numerous infrastructures, industrialization, habitat fragmentation, agricultural expansion and other human interventions. Therefore, there is a great need for conducting a detail floristic study throughout this district.

*Corresponding author, email: robaydakhanam@yahoo.com 
This study has been carried out to construct a taxonomic checklist of the angiosperms under the class Magnoliopsida (dicotyledones) of Narsingdi district based on thorough taxonomic inventories throughout the area to serve as a taxonomic baseline on the current status of the species of this plant group in this area, contribute in further studies on the change in floristic composition, plant species diversity and vegetation, help in adopting appropriate biodiversity conservation initiatives and promote plant resourse-based socioeconomic development projects in this area.

\section{Materials and Methods}

Narsingdi district, comprised of an area of $1140.76 \mathrm{sq} \mathrm{km}$ and and located in between $23^{\circ} 46^{\prime} \mathrm{N}$ and $24^{\circ} 15^{\prime} \mathrm{N}$ and $90^{\circ} 34^{\prime} \mathrm{E}$ and $90^{\circ} 59^{\prime} \mathrm{E}$, is a part of the Dhaka division. It is bordered by Kishoreganj district in the north and northeast, Brahmanbaria district in the east and southeast, Narayanganj district in south and southwest and Gazipur district in the west. This district is consists of six upazilas, namely Belabo, Monohardi, Narsingdi Sadar, Palash, Raipura, and Shibpur. The area is composed of mostly plain lands including numerous agricultural fields, a vast and densely industrial area housing many textile and jute mills etc., a huge fallow lands and homestead areas, many low- and wet lands and some small hills. It includes a total of 89045 hectares of cultivable land and 22154 hectares of fallow land. The maximum annual average temperature in this area is $36^{\circ} \mathrm{C}$, the minimum is $12.7^{\circ} \mathrm{C}$ and the annual rainfall is $2376 \mathrm{~mm}$. The main rivers crossing this district are Meghna, Arial Khan, Haridhoa, Kalagachhia and Paharia.

This study was based on a thorough taxonomic inventory comprised of 32 field trips conducted in different seasons of 2014-2019 throughout Narsingdi district. The collection, processing, drying and preservation of plant specimens were done following standard herbarium methods and techniques (Bridson and Forman, 1989; Singh and Subramaniam, 2008).

The representative plant specimens of all taxa were examined at Plant Systematics and Biodiversity Laboratory of Jahangirnagar University and Bangladesh National Herbarium (DACB). The identification of the specimens was completed consulting taxonomic descriptions and keys available in the relevant literatures (Hooker, 1872-1897; Prain, 1903; Kanjilal et al. 1934, 1938-1940; Nasir and Ali, 1980-2005; Wu and Raven, 1994-2001; Wu et al., 1999-2013), and by matching with the respective voucher specimens of DACB and Jahangirnagar University Herbarium (JUH). The relevant type images available in the web pages of different international herbaria, such the Royal Botanic Gardens Kew (K), and the Conservatoire et Jardin botaniques de la Ville de Genève (G) etc., and illustration of Flora of China (Wu and Raven, 1994-2001; Wu et al., 1999-2013) were also matched. All voucher specimens have been preserved at JUH.

Nomenclatural informations were verified following Flora of China (Wu and Raven, 19942001; Wu et al., 1999-2013), IPNI (2017), The Plant List (2013) and TROPICOS (2017). The common names were collected from Huq (1986), Pasha and Uddin (2013), Ahmed et al. (20082009) and through interviews with the local people. The families have been arranged following Cronquist (1981), and the genera and species under each family alphabetically (Table 1). The economic uses of the species were recorded through interviews with the local people during the field surveys, and consulting the relevant literatures (Ghani, 1998; Van Valkenburg and Bunyapraphatsara, 2002; Ahmed et al., 2008-2009).

The extent of similarities in species composition in six upazilas of the study area was measured by Jaccard coefficient (Jaccard, 1912). Status of threatened plant species listed in Red Data Book of Vascular Plants of Bangladesh (Khan et al., 2001; Ara et al., 2013) was asseesed through field observation and estimation on population size, distribution range and regeneration of each species in the area, collections and consulting the relevent IUCN guidelines (IUCN Standards and Petitions Committee, 2019). 
Table 1. List of the species of Magnoliopsida (dicotyledones) extant in Narsingdi district, Bangladesh.

\begin{tabular}{|c|c|c|c|c|c|c|}
\hline Scientific name & Bangla name & Habit & Habitat & Distribution & Use & RSE \\
\hline \multicolumn{7}{|l|}{ MAGNOLIOPSIDA Brongn. } \\
\hline \multicolumn{7}{|l|}{ ANNONACEAE Juss. } \\
\hline Annona reticulata $\mathrm{L}$. & Nona/Ata & Tree, $s$ & FL, SJ & All upazilas & $\mathrm{Fr}$ & Robayda 2595 \\
\hline A. squamosa $\mathrm{L}$. & Sharifa & Tree, $s$ & $\mathrm{FL}, \mathrm{SJ}$ & All upazilas & Fr & Robayda 2422 \\
\hline $\begin{array}{l}\text { Miliusa velutina (Dunal) Hook. f. \& } \\
\text { Thomson }\end{array}$ & Gandhi Gajari & Tree, $\mathrm{m}$ & SJ & $\mathrm{M}, \mathrm{B}, \mathrm{S}$ & $\mathrm{M}, \mathrm{T}$ & Robayda 2670 \\
\hline Polyalthia longifolia (Sonn.) Thwaites & Debdaru & Tree, 1 & RS & All upazilas & $\mathrm{O}, \mathrm{T}$ & Robayda 697 \\
\hline P. suberosa (Roxb.) Thwaites & Kukuriam & Tree, $s$ & $\mathrm{FL}, \mathrm{SJ}$ & NS, B, M, S & Fr & Robayda 2528 \\
\hline \multicolumn{7}{|l|}{ LAURACEAE Juss. } \\
\hline $\begin{array}{l}\text { Cinnamomum tamala (Buch.-Ham.) T. } \\
\text { Nees \& Nees }\end{array}$ & Tejpata & Tree, $\mathrm{m}$ & FL & $\mathrm{M}, \mathrm{B}, \mathrm{S}$ & $\mathrm{M}, \mathrm{Sp}$ & Robayda 3054 \\
\hline Litsea glutinosa (Lour.) C.B. Rob. & Kukur Chita & Tree, $m$ & $\mathrm{RS}, \mathrm{SJ}$ & All upazilas & $\mathrm{M}, \mathrm{T}$ & Robayda 2082 \\
\hline L. monopetala (Roxb.) Pers. & Bara Kukurchita & Tree, $\mathrm{m}$ & RS, SJ & All upazilas & M & Robayda 2072 \\
\hline Persea americana Mill. & Avocado & Tree, $\mathrm{m}$ & $\mathrm{RS}(\mathrm{pl})$ & $\mathrm{P}$ & $\mathrm{Fr}$ & Robayda 2959 \\
\hline \multicolumn{7}{|l|}{ PIPERACEAE Giseke } \\
\hline Peperomia pellucida (L.) Kunth & Luchi pata & Herb, er & RS, FL & All upazilas & M & Robayda 945 \\
\hline Piper betle $\mathrm{L}$. & Pan & Herb, cl & ML (cu) & M, S, B & M & Robayda 1812 \\
\hline P. longum L. & Pipul morich & Herb, e & RS, FL & NS, B, R, S & M & Robayda 3032 \\
\hline P. sylvaticum Roxb. & Bon Pan & Herb,cl & RS, FL & All upazilas & M & Robayda 1223 \\
\hline \multicolumn{7}{|l|}{ ARISTOLOCHIACEAE Juss. } \\
\hline Aristolochia indica $\mathrm{L}$. & Ishwarmul & Shrub, cl & SJ & $\mathrm{B}, \mathrm{S}, \mathrm{M}$ & M & Robayda 2505 \\
\hline \multicolumn{7}{|l|}{ NELUMBONACEAE A. Rich. } \\
\hline Nelumbo nucifera Gaertn. & Padma & Herb, aq & WL & NS, P, B & M & Robayda 3127 \\
\hline \multicolumn{7}{|l|}{ NYMPHAEACEAE Salisb. } \\
\hline Nymphaea capensis Thunb. & Nil Shapla & Herb, aq & WL & $\mathrm{NS}, \mathrm{B}, \mathrm{M}, \mathrm{R}$ & M & Robayda 2753 \\
\hline N. pubescens Willd. & Shada Shapla & Herb, aq & WL & All upazila & $\mathrm{M}, \mathrm{V}$ & Robayda 2423 \\
\hline N. rubra Roxb. ex Salisb. & Lal Shapla & Herb, aq & WL & $\mathrm{NS}, \mathrm{B}, \mathrm{R}$ & M & Robayda 2751 \\
\hline \multicolumn{7}{|l|}{ RANUNCULACEAE Juss. } \\
\hline Naravelia zeylanica (L.) DC. & Chagul Bati & Herb, cl & SJ & M & - & Robayda 1074 \\
\hline \multicolumn{7}{|l|}{ MENISPERMACEAE Juss. } \\
\hline Stephania japonica (Thunb.) Miers & Akanadi & Herb, cl & $\mathrm{RS}, \mathrm{SJ}$ & All upazilas & M & Robayda 2164 \\
\hline Tiliacora racemosa Colebr. & Bhag-lata & Shrub, cl & SJ, FL & $\mathrm{NS}, \mathrm{B}, \mathrm{M}, \mathrm{S}$ & B \& Th & Robayda 2106 \\
\hline $\begin{array}{l}\text { Tinospora crispa (L.) Hook. f. \& } \\
\text { Thomson }\end{array}$ & Gulancha & Shrub, cl & $\mathrm{SJ}, \mathrm{FL}$ & $\mathrm{B}, \mathrm{S}, \mathrm{M}$ & M & Robayda 2571 \\
\hline T. sinensis (Lour.) Merr. & Padma Gulancha & Shrub, cl & SJ, FL & $\mathrm{B}, \mathrm{NS}, \mathrm{M}$ & M & Robayda 2297 \\
\hline \multicolumn{7}{|l|}{ ULMACEAE Mirb. } \\
\hline Holoptelea integrifolia Planch. & - & Tree, $\mathrm{m}$ & $\mathrm{RS}, \mathrm{SJ}$ & All upazilas & - & Robayda 2171 \\
\hline Trema orientalis (L.) Blume & Jiban & Tree, $\mathrm{s}$ & $\mathrm{RS}, \mathrm{SJ}$ & All upazilas & Fw & Robayda 1365 \\
\hline \multicolumn{7}{|l|}{ MORACEAE Gaudich. } \\
\hline Artocarpus heterophyllus Lam. & Kathal & Tree, 1 & FL & All upazilas & $\mathrm{T}, \mathrm{F}$ & Robayda 60 \\
\hline A. lakoocha Wall. ex Roxb. & Dewa & Tree, $\mathrm{m}$ & SJ & All upazilas & Fr, T, M & Robayda 2268 \\
\hline Ficus benghalensis L. & Bot & Tree, $\mathrm{m}$ & $\mathrm{RS}, \mathrm{SJ}$ & All upazilas & $\mathrm{O}, \mathrm{M}$ & Robayda 1070 \\
\hline F. benjamina $\mathrm{L}$. & Pakur & Tree, $\mathrm{s}$ & $\mathrm{RS}(\mathrm{pl})$ & NS, S & $\mathrm{O}, \mathrm{Fw}$ & Robayda 2450 \\
\hline F. heterophylla L. f. & Bhui Dumur & Shrub & ML, RS & $\mathrm{P}, \mathrm{S}$ & - & Robayda 1611 \\
\hline F. hispida L. f. & Kak Dumur & Tree, $\mathrm{s}$ & SJ, RS & All upazilas & - & Robayda 69 \\
\hline F. pumila $\mathrm{L}$. & Lata Dumur & Herb,cl & $\mathrm{RS}(\mathrm{pl})$ & NS, B, S & $\mathrm{O}$ & Robayda 2787 \\
\hline F. racemosa $\mathrm{L}$. & Jagya Dumur & Tree, $\mathrm{s}$ & ML & All upazilas & $\mathrm{T}$ & Robayda 1523 \\
\hline
\end{tabular}




\begin{tabular}{|c|c|c|c|c|c|c|}
\hline Scientific name & Bangla name & Habit & Habitat & Distribution & Use & RSE \\
\hline F. religiosa $\mathrm{L}$. & Ashwath & Tree, 1 & RS, FL & All upazilas & $\mathrm{T}$ & Robayda 1561 \\
\hline F. rumphii Blume & Gai Ashwath & Tree, 1 & SJ & All upazilas & $\mathrm{T}$ & Robayda 1509 \\
\hline Morus alba $\mathrm{L}$. & Tut & Tree, $\mathrm{m}$ & ML & M & M & Robayda 1266 \\
\hline $\begin{array}{l}\text { Streblus asper Lour. } \\
\text { URTICACEAE Juss. }\end{array}$ & Sheora & Tree, $\mathrm{s}$ & RS, ML & All upazilas & M & Robayda 873 \\
\hline Dendrocnide sinuata (Blume) Chew & Chuchra & Shrub & SJ & B & $\begin{array}{l}\mathrm{Fb}, \mathrm{Fw}, \\
\mathrm{M}\end{array}$ & Robayda 2172 \\
\hline Laportea interrupta (L.) Chew & Lal Bechuti & Herb, er & RS, FL & All upazilas & M & Robayda 1653 \\
\hline Pilea microphylla (L.) Liebm. & Latamaricha & Herb, pr & FL & All upazilas & M & Robayda 1466 \\
\hline $\begin{array}{l}\text { Pouzolzia zeylanica }(\text { L.) Benn. } \\
\text { CASUARINACEAE R. Br. }\end{array}$ & Kullaruki & Herb, er & RS, FL & All upazilas & M & Robayda 1985 \\
\hline $\begin{array}{l}\text { Casuarina equisetifolia } \mathrm{L} . \\
\text { NYCTAGINACEAE Juss. }\end{array}$ & Jhau & Tree, 1 & $\mathrm{RS}(\mathrm{pl})$ & $\mathrm{P}, \mathrm{NS}$ & $\mathrm{O}, \mathrm{T}$ & Robayda 763 \\
\hline Boerhavia diffusa $\mathrm{L}$. & Punarnava & Herb, & RS & All upazilas & M & Robayda 698 \\
\hline Bougainvillea spectabilis Willd. & Baganbilash & Shrub, cl & $\mathrm{FL}(\mathrm{pl})$ & All upazilas & $\mathrm{O}$ & Robayda 1939 \\
\hline Mirabilis jalapa $\mathrm{L}$. & Sandhya Maloti & Herb, er & RS & All upazilas & M & Robayda 346 \\
\hline $\begin{array}{l}\text { CACTACEAE Juss. } \\
\text { Opuntia dillenii (Ker Gawl.) Haw. } \\
\text { AMARANTHACEAE Juss. }\end{array}$ & Phanimanasa & Herb, er & FL (Pl) & All upazilas & $\mathrm{O}, \mathrm{M}$ & Robayda 2922 \\
\hline Achyranthes aspera $\mathrm{L}$. & Apang & Herb, er & RS, ML & All upazilas & - & Robayda 2152 \\
\hline Alternanthera paronychioides A. St.-Hil. & Jhuli Khata & Herb, pr & RS, FL & $\mathrm{NS}, \mathrm{B}, \mathrm{S}$ & - & Robayda 3043 \\
\hline A. philoxeroides (Mart.) Griseb. & Malancha, Henchi & Herb, pr & WL, ML & All upazilas & $\mathrm{V}$ & Robayda 881 \\
\hline A. sessilis (L.) R. Br. ex DC. & Sachi shak & Herb, pr & RS, ML & All upazilas & M & Robayda 2568 \\
\hline Amaranthus lividus $\mathrm{L}$. & Gobura Notey & Herb, er & FL (cu) & All upazilas & $\mathrm{V}$ & Robayda 281 \\
\hline A. spinosus $\mathrm{L}$. & Kata Nate & Herb, er & RS & All upazilas & M & Robayda 1661 \\
\hline A. tricolor $\mathrm{L}$. & Data & Herb, er & FL (cu) & All upazilas & $\mathrm{V}$ & Robayda 3137 \\
\hline A. viridis $\mathrm{L}$. & Nate Shak & Herb, er & RS, FL & All upazilas & $\mathrm{V}, \mathrm{M}$ & Robayda 1413 \\
\hline Celosia cristata $\mathrm{L}$. & Moragphul & Herb, er & FL & $\mathrm{B}, \mathrm{S}, \mathrm{M}, \mathrm{P}$ & $\mathrm{O}, \mathrm{M}$ & Robayda 2750 \\
\hline Chenopodium album $\mathrm{L}$. & Batua shak & Herb, er & $\mathrm{AF}$ & All upazilas & $\mathrm{V}, \mathrm{M}$ & Robayda 1467 \\
\hline C. ambrosioides $\mathrm{L}$. & Chandan Betu & Herb, er & ML & NS, B & M & Robayda 2017 \\
\hline Cyathula prostrata (L.) Blume & Chaya Apang & Herb, pr & RS & All upazilas & M & Robayda 1286 \\
\hline Deeringia amaranthoides (Lam.) Merr. & Golamohani & Herb, sc & FL & $\mathrm{R}$ & - & Robayda 3084 \\
\hline Gomphrena celosioides Mart. & - & Herb, pr & RS, FL & $\mathrm{R}, \mathrm{B}$ & - & Robayda 1984 \\
\hline $\begin{array}{l}\text { G. globosa } \mathrm{L} \text {. } \\
\text { PORTULACACEAE Juss. }\end{array}$ & Botamphul & Herb, er & RS & NS, B & $\mathrm{O}, \mathrm{M}$ & Robayda 2749 \\
\hline Portulaca oleracea $\mathrm{L}$. & Lunia Shak & Herb, pr & RS, FL & All upazilas & $\mathrm{V}$ & Robayda 2137 \\
\hline P. quadrifida $\mathrm{L}$. & $\begin{array}{l}\text { Chhoto Lunia } \\
\text { Shak }\end{array}$ & Herb, pr & FL & $\mathrm{B}, \mathrm{S}$ & $\mathrm{V}$ & Robayda 2353 \\
\hline $\begin{array}{l}\text { P. grandiflora Hook. } \\
\text { BASELLACEAE Raf. }\end{array}$ & Porchulaca & Herb, pr & RS & All upazilas & $\mathrm{O}$ & Robayda 2508 \\
\hline $\begin{array}{l}\text { Basella rubra L. } \\
\text { MOLLUGINACEAE Baril. }\end{array}$ & Pui Shak & Herb, cl & FL & All upazilas & $\mathrm{V}$ & Robayda 1667 \\
\hline $\begin{array}{l}\text { Glinus oppositifolius (L.) Aug. DC. } \\
\text { CARYOPHYLLACEAE Juss. }\end{array}$ & Gema Shak & Herb, pr & RS, FL & $\mathrm{M}, \mathrm{B}, \mathrm{P}$ & $\mathrm{V}$ & Robayda 1875 \\
\hline $\begin{array}{l}\text { Polycarpon prostratum (Forssk.) Asch. } \\
\& \text { Schweinf. ex Asch. }\end{array}$ & Ghima & Herb, pr & ML & NS, B, P & - & Robayda 2033 \\
\hline $\begin{array}{l}\text { Stellaria media (L.) Vill. } \\
\text { POLYGONACEAE Juss. }\end{array}$ & Sada Phulki, Tara & Herb, er & FL & M, B & - & Robayda 1809 \\
\hline Persicaria assamica (Meisn.) Soják & Assami Bishkatali & Herb, er & ML & NS & - & Robayda 2015 \\
\hline
\end{tabular}




\begin{tabular}{|c|c|c|c|c|c|c|}
\hline Scientific name & Bangla name & Habit & Habitat & Distribution & Use & RSE \\
\hline P. chinensis (L.) H. Gross & Mohicharan Shak & Herb, er & $\mathrm{RS}$ & $\mathrm{B}, \mathrm{M}$ & - & Robayda 2914 \\
\hline P. hydropiper (L.) Delarbre & Bishkatali & Herb, er & ML & All upazilas & M & Robayda 2678 \\
\hline P. lanata (Roxb.) Tzvelev & Shet Panimorich & Herb, er & ML & $\mathrm{P}, \mathrm{B}$ & - & Robayda 1621 \\
\hline P. lapathifolia (L.) Delarbre & Panimorich & Herb, er & ML & $\mathrm{B}, \mathrm{P}, \mathrm{S}$ & M & Robayda 3028 \\
\hline P. minor (Huds.) Opiz & Chhoto Bishkatali & Herb, er & ML & $\mathrm{NS}, \mathrm{P}, \mathrm{B}, \mathrm{S}$ & - & Robayda 1169 \\
\hline P. orientalis (L.) Spach & Baro Panimorich & Herb, er & WL & All upazilas & M & Robayda 2482 \\
\hline Polygonum effusum Meisn. & Raniphul & Herb, pr & ML & $\mathrm{NS}, \mathrm{R}, \mathrm{B}$ & M & Robayda 3013 \\
\hline P. plebeium $\mathrm{R}$. Br. & Khudi Bishkatali & Herb, er & FL & $\mathrm{M}, \mathrm{P}, \mathrm{R}$ & $\mathrm{V}$ & Robayda 1671 \\
\hline P. pubescens Blume & Lal-Bishkatali & Herb, er & FL & NS, S & - & Robayda 2949 \\
\hline Rumex maritimus L. & Bon Palong & Herb, er & ML & All upazilas & M & Robayda 1806 \\
\hline \multicolumn{7}{|l|}{ DILLENIACEAE Salisb. } \\
\hline Dillenia indica $\mathrm{L}$. & Chalta & Tree, $\mathrm{m}$ & SJ & All upazilas & $\mathrm{Fr}$ & Robayda 1115 \\
\hline \multicolumn{7}{|l|}{ DIPTEROCARPACEAE Blume } \\
\hline Dipterocarpus turbinatus Gaertn. & Garjan & Tree, 1 & Wd & $\mathrm{S}$ & $\mathrm{T}$ & Robayda 134 \\
\hline \multicolumn{7}{|l|}{ CLUSIACEAE Lindl. } \\
\hline Calophyllum inophyllum $\mathrm{L}$. & Sultan Champa & Tree, 1 & $\mathrm{RS}(\mathrm{pl})$ & NS & $\mathrm{O}$ & Robayda 2964 \\
\hline Garcinia cowa Roxb. & Kau & Tree, 1 & SJ & $\mathrm{S}$ & Fr & Robayda 2657 \\
\hline \multicolumn{7}{|l|}{ ELAEOCARPACEAE Juss. } \\
\hline Elaeocarpus floribundus Blume & Jalpai & Tree, $\mathrm{m}$ & $\mathrm{RS}$ & All upazilas & $\mathrm{Fr}$ & Robayda 2313 \\
\hline E. lanceifolius Roxb. & - & Tree, $\mathrm{m}$ & FL & B & - & Robayda 2546 \\
\hline \multicolumn{7}{|l|}{ TILIACEAE Juss. } \\
\hline Corchorus aestuans $\mathrm{L}$. & Banpat & Herb, pr & FL & $\mathrm{P}$ & M & Robayda 759 \\
\hline C. capsularis $\mathrm{L}$. & Deshi-pat & Shrub & $\mathrm{RS}$ & All upazilas & $\mathrm{Fb}$ & Robayda 2059 \\
\hline Grewia nervosa (Lour.) Panigrahi & Pichandi, Datoi & Tree, $\mathrm{s}$ & $\mathrm{RS}, \mathrm{SJ}$ & All upazilas & Fw & Robayda 1969 \\
\hline Triumfetta rhomboidea Jacq. & Bon Okra & Shrub & $\mathrm{RS}$ & All upazilas & M & Robayda 840 \\
\hline \multicolumn{7}{|l|}{ STERCULIACEAE Vent. } \\
\hline Abroma augusta (L.) L. f. & Ulatkambal & Shrub, & FL & $\mathrm{M}, \mathrm{B}, \mathrm{S}$ & M & Robayda 2786 \\
\hline Melochia corchorifolia $\mathrm{L}$. & Tiki Okra & Herb, er & RS & All upazilas & W & Robayda 2006 \\
\hline Pentapetes phoenicea $\mathrm{L}$. & Bandhuli & Shrub & $\mathrm{RS}$ & NS, S & $\mathrm{O}$ & Robayda 2784 \\
\hline \multicolumn{7}{|l|}{ MALVACEAE Juss. } \\
\hline Abelmoschus esculentus (L.) Moench & Dheros & Herb, er & ML (cu) & All upazilas & $\mathrm{V}$ & Robayda 1220 \\
\hline A. moschatus Medik. & Mushak-dana & Shrub & RS, FL & $\mathrm{B}, \mathrm{S}$ & M & Robayda 2431 \\
\hline Abutilon theophrasti Medik. & American Pat & Shrub & $\mathrm{RS}$ & NS, P, S, B & $\mathrm{Fb}$ & Robayda 2469 \\
\hline Bombax ceiba $\mathrm{L}$. & Shimul & Tree, 1 & RS, FL & $\mathrm{B}, \mathrm{R}, \mathrm{S}, \mathrm{P}$ & $\mathrm{T}, \mathrm{Fb}$ & Robayda 1690 \\
\hline Ceiba pentandra (L.) Gaertn. & Swet Tula, Kapok & Tree, 1 & $\mathrm{RS}$ & NS & $\mathrm{T}, \mathrm{Fb}$ & Robayda 3036 \\
\hline Fioria vitifolia (L.) Mattei & Ban Carpas & Herb, er & RS, FL & $\mathrm{P}, \mathrm{M}, \mathrm{B}$ & $\mathrm{Fb}$ & Robayda 1423 \\
\hline Hibiscus rosa-sinensis $\mathrm{L}$. & Jaba & Shrub & $\mathrm{RS}$ & All upazilas & $\mathrm{O}$ & Robayda 1370 \\
\hline H. sabdariffa $\mathrm{L}$. & Mesta Pat & Herb, er & ML & $\mathrm{B}, \mathrm{S}, \mathrm{M}, \mathrm{NS}$ & $\mathrm{Fb}$ & Robayda 2238 \\
\hline H. surattensis $\mathrm{L}$. & - & Herb, er & ML & $\mathrm{P}$ & $\mathrm{Fb}, \mathrm{M}$ & Robayda 3014 \\
\hline Malvaviscus arboreus Cav. & Morich jaba & Shrub & $\mathrm{RS}$ & All upazilas & $\mathrm{O}$ & Robayda 92 \\
\hline Sida acuta Burm. f. & Berela & Herb, er & $\mathrm{RS}$ & All upazilas & - & Robayda 265 \\
\hline S. cordata (Burm. f.) Borss. Waalk. & Junka & Herb, er & FL & $\mathrm{R}, \mathrm{S}$ & - & Robayda 878 \\
\hline S. cordifolia $\mathrm{L}$. & Berela & Herb, er & RS & $\mathrm{R}, \mathrm{S}, \mathrm{M}$ & $\mathrm{Fb}$ & Robayda 2368 \\
\hline S. rhombifolia $\mathrm{L}$. & Lal berela & Herb, er & $\mathrm{RS}$ & All upazilas & M & Robayda 1991 \\
\hline $\begin{array}{l}\text { Thespesia lampas (Cav.) Dalzell \& A. } \\
\text { Gibson }\end{array}$ & Ban Karpas & Shrub & SJ & M, B & $\mathrm{Fb}$ & Robayda 1075 \\
\hline Urena lobata $\mathrm{L}$. & Ban okra & Shrub & $\mathrm{RS}$ & All upazilas & $\mathrm{Fb}$ & Robayda 1988 \\
\hline \multicolumn{7}{|l|}{ LECYTHIDACEAE A. Rich. } \\
\hline Barringtonia acutangula (L.) Gaertn. & Hijal & Tree, $\mathrm{m}$ & ML & $\mathrm{NS}, \mathrm{B}, \mathrm{S}$ & M & Robayda 2375 \\
\hline
\end{tabular}




\begin{tabular}{|c|c|c|c|c|c|c|}
\hline Scientific name & Bangla name & Habit & Habitat & Distribution & Use & RSE \\
\hline \multicolumn{7}{|l|}{ FLACOURTIACEAE Rich. ex DC. } \\
\hline Flacourtia indica (Burm. f.) Merr. & Beuchi & Shrub & SJ & $\mathrm{NS}, \mathrm{S}, \mathrm{M}$ & M & Robayda 08 \\
\hline $\begin{array}{l}\text { F. jangomas (Lour.) Raeusch. } \\
\text { VIOLACEAE Batsch }\end{array}$ & Paniala, Lukluki & Tree, $\mathrm{s}$ & SJ & $\mathrm{B}, \mathrm{M}, \mathrm{S}$ & Fr. M, T & Robayda 2245 \\
\hline $\begin{array}{l}\text { Hybanthus enneaspermus (L.) F. Muell. } \\
\text { CARICACEAE Dumort. }\end{array}$ & Nunbora & Herb, er & FL & $\mathrm{R}$ & M & Robayda 1947 \\
\hline $\begin{array}{l}\text { Carica papaya } \mathrm{L} . \\
\text { CUCURBITACEAE Juss. }\end{array}$ & Pepe & Tree, $\mathrm{s}$ & ML & All upazilas & $\mathrm{V}, \mathrm{Fr}, \mathrm{M}$ & Robayda 1505 \\
\hline Benincasa hispida (Thunb.) Cogn. & Chalkumra & Herb, cl & ML & All upazilas & $\mathrm{V}$ & Robayda 2083 \\
\hline $\begin{array}{l}\text { Citrullus lanatus (Thunb.) Matsum. \& } \\
\text { Nakai }\end{array}$ & Tormuj & Herb, cl & RS & $\mathrm{NS}, \mathrm{R}$ & Fr, M & Robayda 3022 \\
\hline Coccinia grandis (L.) Voigt & Telakucha & Herb, cl & $\mathrm{RS}, \mathrm{SJ}$ & All upazilas & $\mathrm{V}, \mathrm{M}$ & Robayda 2271 \\
\hline Cucumis collosus (Rottler) Cogn. & Tita bangi & Herb, cl & RS & $\mathrm{B}, \mathrm{M}$ & - & Robayda 2604 \\
\hline C. melo $\mathrm{L}$. & Bangi/Futi & Herb, cl & RS & NS, R, M & $\mathrm{Fr}, \mathrm{M}$ & Robayda 2604 \\
\hline C. sativus $\mathrm{L}$. & Shasa & Herb, cl & FL (cu) & All upazilas & $\mathrm{V}$ & Robayda 2084 \\
\hline Cucurbita maxima Duchesne & Misti Kumra & Herb, cl & $\mathrm{RS}(\mathrm{cu})$ & All upazilas & $\mathrm{V}$ & Robayda 1646 \\
\hline $\begin{array}{l}\text { Gymnopetalum cochinchinense (Lour.) } \\
\text { Kurz }\end{array}$ & Ban Patol & Herb, cl & RS, SJ & All upazilas & - & Robayda 2217 \\
\hline Lagenaria siceraria (Molina) Standl. & $\mathrm{Lau} / \mathrm{Kadu}$ & Herb, cl & ML (cu) & All upazilas & $\mathrm{V}$ & Robayda 1582 \\
\hline Luffa acutangula (L.) Roxb. & Zhinga & Herb, cl & FL (cu) & All upazilas & $\mathrm{V}$ & Robayda 2300 \\
\hline L. cylindrica M. Roem. & Dhundal & Herb, cl & SJ & All upazilas & $\mathrm{V}$ & Robayda 2557 \\
\hline Momordica charantia $\mathrm{L}$. & Uchchhey/Korolla & Herb, cl & $\mathrm{RS}(\mathrm{cu})$ & All upazilas & $\mathrm{V}$ & Robayda 1597 \\
\hline M. cochinchinensis (Lour.) Spreng. & Kakrol & Herb, cl & $\mathrm{SJ}(\mathrm{cu})$ & All upazilas & $\mathrm{V}$ & Robayda 1662 \\
\hline M. dioica Roxb. ex Willd. & Dharkorolla & Herb, cl & SJ & B & $\mathrm{V}, \mathrm{M}$ & Robayda 1294 \\
\hline Mukia maderaspatana (L.) M. Roem. & Agmukhi, Bilari & Herb, cl & RS & $\mathrm{B}, \mathrm{M}, \mathrm{S}$ & M & Robayda 2475 \\
\hline Solena amplexicaulis (Lam.) Gandhi & Rakhal Shasha & Herb, cl & SJ & B & M & Robayda 2379 \\
\hline Trichosanthes anguina $\mathrm{L}$. & Chichinga & Herb, cl & $\mathrm{SJ}(\mathrm{cu})$ & All upazilas & $\mathrm{V}$ & Robayda 2005 \\
\hline T. cordata Roxb. & Bhuikakra & Herb, cl & SJ & $\mathrm{B}, \mathrm{M}$ & M & Robayda 2155 \\
\hline T. dioica Roxb. & Patal & Herb, cl & $\mathrm{SJ}(\mathrm{cu})$ & S, M, B & M & Robayda 1747 \\
\hline T. tricuspidata Lour. & Makal & Herb, cl & SJ & $\mathrm{B}, \mathrm{S}, \mathrm{R}$ & - & Robayda 2554 \\
\hline CAPPARACEAE Juss. & & & & & & \\
\hline Capparis spinosa $\mathrm{L}$. & Kabia, Katai & Shrub, cl & SJ & M, S & $\mathrm{Fd}, \mathrm{M}$ & Robayda 852 \\
\hline Cleome rutidosperma DC. & Beguni Hurhure & Herb, dc & RS, FL & All upazilas & - & Robayda 1920 \\
\hline C. viscosa $\mathrm{L}$. & Halud Hurhure & Herb, er & RS, FL & All upazilas & - & Robayda 2239 \\
\hline Crateva magna (Lour.) DC. & $\begin{array}{l}\text { Tikthashak, } \\
\text { Bonnya }\end{array}$ & Tree, m & ML & $\begin{array}{l}\text { NS, B, R, M, } \\
\text { S }\end{array}$ & - & Robayda 1974 \\
\hline BRASSICACEAE Burnett & & & & & & \\
\hline Brassica juncea (L.) Czern. & Rai sarisha & Herb, er & RS & $\mathrm{S}, \mathrm{B}, \mathrm{R}$ & $\mathrm{Ol}$ & Robayda 581 \\
\hline B. napus L. & Sarisha & Herb, er & $\mathrm{RS}(\mathrm{cu})$ & All upazilas & $\mathrm{Ol}$ & Robayda 1515 \\
\hline B. rapa $\mathrm{L}$. & Shalgam & Herb, er & $\mathrm{RS}(\mathrm{cu})$ & All upazilas & $\mathrm{V}$ & Robayda 1545 \\
\hline Cardamine flexuosa With. & - & Herb, dc & $\mathrm{AF}, \mathrm{RS}$ & $\mathrm{P}, \mathrm{S}, \mathrm{R}, \mathrm{NS}$ & - & Robayda 1506 \\
\hline Raphanus sativus L. & Mula & Herb, er & ML (cu) & All upazilas & $\mathrm{V}, \mathrm{M}$ & Robayda 1209 \\
\hline $\begin{array}{l}\text { Rorippa palustris (L.) Besser } \\
\text { MORINGACEAE Martinov }\end{array}$ & Panisarisha & Herb, dc & RS, FL & $\mathrm{P}, \mathrm{R}, \mathrm{S}, \mathrm{M}$ & - & Robayda 334 \\
\hline $\begin{array}{l}\text { Moringa ovalifolia Dinter \& Berger } \\
\text { SAPOTACEAE Juss. }\end{array}$ & Sajna & Tree, $\mathrm{m}$ & RS, FL & All upazilas & $\mathrm{V}$ & Robayda 1654 \\
\hline Chrysophyllum cainito L. & Star Apple & Tree, $\mathrm{m}$ & $\mathrm{RS}(\mathrm{pl})$ & $\mathrm{R}$ & $\mathrm{O}$ & Robayda 2384 \\
\hline $\begin{array}{l}\text { Madhuca longifolia (J. Koenig ex L.) J.F. } \\
\text { Macbr. }\end{array}$ & Mahua & Tree, 1 & $\mathrm{FL}, \mathrm{SJ}$ & $\mathrm{B}, \mathrm{S}$ & M & Robayda 2733 \\
\hline
\end{tabular}




\begin{tabular}{|c|c|c|c|c|c|c|}
\hline Scientific name & Bangla name & Habit & Habitat & Distribution & Use & RSE \\
\hline Mimusops elengi $\mathrm{L}$. & Bakul & Tree, 1 & $\mathrm{RS}(\mathrm{pl})$ & $\mathrm{P}, \mathrm{NS}, \mathrm{M}$ & $\mathrm{O}, \mathrm{T}$ & Robayda 787 \\
\hline \multicolumn{7}{|l|}{ EBENACEAE Gürke } \\
\hline Diospyros blancoi A. DC. & Beelati Gab & Tree, $\mathrm{m}$ & SJ & $\mathrm{B}, \mathrm{P}, \mathrm{NS}, \mathrm{M}$ & Fr, $\mathrm{T}$ & Robayda 2737 \\
\hline D. malabarica (Desr.) Kostel. & Deshi Gab & Tree, 1 & SJ & $\mathrm{B}, \mathrm{S}, \mathrm{M}, \mathrm{NS}$ & Fr, T & Robayda 2138 \\
\hline D. montana Roxb. & Tamal & Tree, $\mathrm{s}$ & SJ & $\mathrm{P}, \mathrm{B}, \mathrm{R}$ & $\mathrm{T}$ & Robayda 2251 \\
\hline \multicolumn{7}{|l|}{ PRIMULACEAE Batsch $e x$ Borkh. } \\
\hline Ardisia humilis Vahl & Chauldhoa & Shrub & SJ & $\mathrm{B}, \mathrm{S}, \mathrm{P}$ & - & Robayda 1731 \\
\hline \multicolumn{7}{|l|}{ CRASSULACEAE J.St.-Hil. } \\
\hline $\begin{array}{l}\text { Kalanchoe blossfeldiana Poelln. } \\
\text { ROSACEAE Juss. }\end{array}$ & Patharkuchi & Herb, er & RS & $\mathrm{B}, \mathrm{S}, \mathrm{NS}, \mathrm{P}$ & $\mathrm{O}$ & Robayda 944 \\
\hline $\begin{array}{l}\text { Rosa chinensis Jacq. } \\
\text { FABACEAE Lindl. }\end{array}$ & Kanta Golap & \multicolumn{4}{|c|}{ FABACEAE Lindl. } & Robayda 2752 \\
\hline $\begin{array}{l}\text { Acacia auriculiformis A. Cunn. ex } \\
\text { Benth. }\end{array}$ & Akashmoni & Tree, 1 & Wd & All upazilas & $\mathrm{T}$ & Robayda 940 \\
\hline A. longifolia (Andrews) Willd. & Hybrid Acacia & Tree, $\mathrm{s}$ & Wd & $\mathrm{B}, \mathrm{NS}, \mathrm{P}, \mathrm{R}$ & $\mathrm{T}$ & Robayda 2956 \\
\hline A. mangium Willd. & Mangium & Tree, 1 & Wd & All upazilas & $\mathrm{T}$ & Robayda 2957 \\
\hline A. nilotica (L.) Willd. ex Delile & Babla & Tree, $\mathrm{m}$ & RS & M & $\mathrm{T}$ & Robayda 1090 \\
\hline Aeschynomene indica $\mathrm{L}$. & Bhatsola & Shrub & RS, FL & NS, P, B, R & - & Robayda 2716 \\
\hline Albizia lebbeck (L.) Benth. & Siris, Kalo Karoi & Tree, 1 & RS & All upazilas & $\mathrm{T}$ & Robayda 2097 \\
\hline $\begin{array}{l}\text { A. lucidior (Steud.) I.C. Nielsen ex H. } \\
\text { Hara }\end{array}$ & Sil-Kioroi & Tree, 1 & RS & $\mathrm{B}, \mathrm{S}, \mathrm{P}$ & $\mathrm{T}$ & Robayda 2919 \\
\hline A. richardiana King \& Prain & Gagan Sirish & Tree, 1 & RS & All upazilas & $\mathrm{T}$ & Robayda 1471 \\
\hline Alysicarpus rugosus (Willd.) DC. & - & Herb, dc & FL & $\mathrm{P}, \mathrm{B}, \mathrm{NS}, \mathrm{M}$ & - & Robayda 1832 \\
\hline Arachis hypogaea $\mathrm{L}$. & Cheena Badam & Herb, dc & $\mathrm{AF}$ & $\mathrm{P}, \mathrm{NS}, \mathrm{R}$ & $\mathrm{Ol}$ & Robayda 2039 \\
\hline Bauhinia acuminata $\mathrm{L}$. & Sada Kanchon & Tree, $\mathrm{s}$ & RS & All upazilas & $\mathrm{O}$ & Robayda 108 \\
\hline Butea monosperma (Lam.) Taub. & Palash & Tree, $\mathrm{m}$ & SJ & $\mathrm{P}, \mathrm{NS}, \mathrm{S}$ & $\mathrm{O}, \mathrm{M}$ & Robayda 2430 \\
\hline Caesalpinia bonduc (L.) Roxb. & Nata/Jhagragota & Shrub & $\mathrm{RS}, \mathrm{SJ}$ & $\mathrm{R}$ & M & Robayda 3033 \\
\hline C. pulcherrima (L.) Sw. & Radhachura & Shrub & RS & NS, S, P & $\mathrm{O}, \mathrm{M}$ & Robayda 2462 \\
\hline Cajanus cajan (L.) Huth & Arahar & Shrub & RS & All upazilas & $\mathrm{Pu}, \mathrm{M}$ & Robayda 1475 \\
\hline Calliandra surinamensis Benth. & $\begin{array}{l}\text { Surinam powderd } \\
\text { puff }\end{array}$ & Tree, $\mathrm{s}$ & RS & NS, S, P & $\mathrm{O}$ & Robayda 564 \\
\hline Canavalia virosa (Roxb.) Wight \& Arn. & Kath Shim & Shrub & SJ & $\mathrm{B}, \mathrm{P}, \mathrm{M}, \mathrm{R}$ & $\mathrm{V}$ & Robayda 2798 \\
\hline Cassia fistula $\mathrm{L}$. & Sonalu, Badarlathi & Tree, $\mathrm{m}$ & RS & All upazilas & $\mathrm{T}, \mathrm{M}$ & Robayda 1997 \\
\hline Cicer arietinum $\mathrm{L}$. & Chhola & Herb, er & $\mathrm{AF}$ & $\begin{array}{l}\mathrm{R}, \mathrm{B}, \mathrm{NS}, \mathrm{M}, \\
\mathrm{S}\end{array}$ & $\mathrm{Pu}$ & Robayda 3129 \\
\hline Clitoria ternatea $\mathrm{L}$. & Aparajita & Herb, cl & FL & All upazilas & $\mathrm{O}$ & Robayda 671 \\
\hline Crotalaria pallida Aiton & Jhunjhuni & Herb, er & RS, FL & All upazilas & - & Robayda 417 \\
\hline Dalbergia sissoo Roxb. ex DC. & Sissoo Gachh & Tree, 1 & RS & All upazilas & $\mathrm{T}$ & Robayda 2899 \\
\hline D. stipulacea Roxb. & Dadbari & Tree, $\mathrm{s}$ & RS & S & & Robayda 3055 \\
\hline Delonix regia (Bojer ex Hook.) Raf. & Krishnachura & Tree, 1 & RS & All Upazilas & $\mathrm{O}$ & Robayda 2687 \\
\hline Derris monticola Prain & - & Shrub & SJ & NS & - & Robayda 2809 \\
\hline Desmodium gangeticum (L.) DC. & Salpani & Shrub & RS, FL & $\mathrm{NS}, \mathrm{P}, \mathrm{R}, \mathrm{S}$ & M & Robayda 1712 \\
\hline D. gyroides (Roxb. ex Link) DC. & - & Shrub & RS & $\mathrm{NS}, \mathrm{P}, \mathrm{B}, \mathrm{M}$ & - & Robayda 582 \\
\hline D. triflorum (L.) DC. & Kulalia & Herb, pr & RS, FL & All upazilas & - & Robayda 1943 \\
\hline Erythrina fusca Lour. & Kanta Mander & Tree, $\mathrm{m}$ & $\mathrm{RS}, \mathrm{SJ}$ & $\mathrm{NS}, \mathrm{B}, \mathrm{S}, \mathrm{M}$ & Fw, M & Robayda 1884 \\
\hline E. variegata $\mathrm{L}$. & Madar & Tree, $\mathrm{m}$ & $\mathrm{RS}, \mathrm{SJ}$ & $\mathrm{B}, \mathrm{M}, \mathrm{S}, \mathrm{NS}$ & Fw & Robayda 2396 \\
\hline Lablab purpureus (L.) Sweet & Sheem & Herb, cl & FL & All upazilas & $\mathrm{V}, \mathrm{Pu}$ & Robayda 247 \\
\hline Lathyrus sativus L. & Khesari & Herb, dc & ML & All upazilas & $\mathrm{Pu}, \mathrm{Fd}$ & Robayda 1593 \\
\hline Leucaena leucocephala (Lam.) de Wit & Ipil-Ipil & Tree, $\mathrm{m}$ & RS & All upazilas & $\mathrm{O}, \mathrm{Fd}$ & Robayda 1262 \\
\hline
\end{tabular}




\begin{tabular}{|c|c|c|c|c|c|c|}
\hline Scientific name & Bangla name & Habit & Habitat & Distribution & Use & RSE \\
\hline Mimosa pudica $\mathrm{L}$. & Lajjabati & Herb, er & RS, FL & All upazilas & M & Robayda 1069 \\
\hline Mucuna pruriens (L.) DC. & Al-kushi & Herb, cl & RS & $\mathrm{P}$ & M & Robayda 1758 \\
\hline $\begin{array}{l}\text { Peltophorum pterocarpum (DC.) Backer } \\
\text { ex K. Heyne }\end{array}$ & $\begin{array}{l}\text { Halud } \\
\text { Krishnachura, } \\
\text { Kanakchura }\end{array}$ & Tree, 1 & $\mathrm{RS}$ & NS, S, P & $\mathrm{O}$ & Robayda 2715 \\
\hline Phaseolus vulgaris $\mathrm{L}$. & Farash Bean & Herb, er & ML (cu) & $\mathrm{P}, \mathrm{S}, \mathrm{B}, \mathrm{M}$ & $\mathrm{V}$ & Robayda 330 \\
\hline Pueraria phaseoloides (Roxb.) Benth. & - & Herb, cl & $\mathrm{RS}$ & $\mathrm{P}, \mathrm{NS}, \mathrm{B}$ & - & Robayda 1498 \\
\hline Rhynchosia minima (L.) DC. & - & Herb, cl & FL & $\mathrm{P}$ & - & Robayda 271 \\
\hline Samanea saman (Jacq.) Merr. & Randi Koroi & Tree, 1 & $\mathrm{RS}$ & All upazilas & $\mathrm{O}, \mathrm{T}$ & Robayda 1066 \\
\hline Senna alata (L.) Roxb. & Dadmardan & Shrub & $\mathrm{RS}, \mathrm{SJ}$ & $\mathrm{NS}, \mathrm{P}, \mathrm{S}, \mathrm{M}$ & M & Robayda 2771 \\
\hline S. occidentalis (L.) Link & Bara Kalkasunda & Herb, er & RS & All upazilas & M & Robayda 385 \\
\hline S. sophera (L.) Roxb. & Bara Kalkasunda & Shrub & $\mathrm{RS}$ & NS, R & M & Robayda 2945 \\
\hline S. tora (L.) Roxb. & $\begin{array}{l}\text { Chhoto } \\
\text { Kalkasunda }\end{array}$ & Shrub & RS, FL & All upazilas & M & Robayda 568 \\
\hline Sesbania bispinosa (Jacq.) W. Wight & Dhaincha & Shrub & ML & All upazilas & $\mathrm{Fd}, \mathrm{Fw}$ & Robayda 2816 \\
\hline S. sesban (L.) Merr. & Jyonti & Shrub & ML & All upazilas & $\mathrm{Fd}$ & Robayda 1376 \\
\hline Spatholobus acuminatus Benth. & Palashi lata & Shrub, cl & SJ & $S$ & - & Robayda 113 \\
\hline Tamarindus indica $\mathrm{L}$. & Tetul & Tree, 1 & RS & All upazilas & Fr, M & Robayda 1431 \\
\hline Uraria lagopus DC. & - & Herb, er & FL & $\mathrm{S}, \mathrm{B}, \mathrm{M}$ & - & Robayda 1909 \\
\hline Vicia hirsuta (L.) Gray & Masurechana & Herb, cl & $\mathrm{RS}$ & $\mathrm{P}$ & $\mathrm{Fd}$ & Robayda 1464 \\
\hline Vigna marina (Burm.) Merr. & Nonta Shim & Herb, cl & ML & $\mathrm{P}$ & - & Robayda 2992 \\
\hline V. mungo (L.) Hepper & Mash Kalai & Herb, er & ML (cu) & $\mathrm{B}, \mathrm{M}, \mathrm{S}, \mathrm{R}$ & & Robayda 1752 \\
\hline V. unguiculata (L.) Walp. & Borboti & Herb, cl & FL & All upazilas & $\mathrm{V}, \mathrm{Pu}$ & Robayda 2522 \\
\hline \multicolumn{7}{|l|}{ HALORAGACEAE R. Br. } \\
\hline $\begin{array}{l}\text { Myriophyllum tetrandrum Roxb. } \\
\text { LYTHRACEAE J.St.-Hil. }\end{array}$ & - & Herb, aq & WL & NS, B, P & - & Robayda 178 \\
\hline Cuphea hyssopifolia Kunth & - & Herb, er & $\mathrm{FL}(\mathrm{pl})$ & $\mathrm{B}, \mathrm{M}, \mathrm{S}, \mathrm{NS}$ & $\mathrm{O}$ & Robayda 2524 \\
\hline Lagerstroemia speciosa (L.) Pers. & Jarul & Tree, $\mathrm{m}$ & $\mathrm{RS}$ & $\mathrm{NS}, \mathrm{P}$ & $\mathrm{O}$ & Robayda 2447 \\
\hline L. indica $\mathrm{L}$. & Cheery & Tree, $\mathrm{s}$ & $\mathrm{RS}$ & NS, S, P & $\mathrm{O}$ & Robayda 463 \\
\hline Lawsonia inermis L. & Mehedi & Shrub & FL & All upazilas & Dy & Robayda 2512 \\
\hline Rotala indica (Willd.) Koehne & - & Herb, aq & WL & $\mathrm{NS}, \mathrm{S}$ & - & Robayda 1158 \\
\hline $\begin{array}{l}\text { R. rotundifolia (Buch.-Ham. ex Roxb.) } \\
\text { Koehne }\end{array}$ & - & Herb, er & WL & All upazilas & - & Robayda 1891 \\
\hline \multicolumn{7}{|l|}{ MYRTACEAE Juss. } \\
\hline Callistemon citrinus (Curtis) Skeels & Bottle Brush & Tree, $\mathrm{s}$ & $\mathrm{RS}(\mathrm{pl})$ & $\mathrm{P}, \mathrm{NS}$ & $\mathrm{O}$ & Robayda 686 \\
\hline Eucalyptus camaldulensis Dehnh. & Eucalyptus & Tree, 1 & $\begin{array}{l}\mathrm{RS}, \mathrm{Wd} \\
(\mathrm{pl})\end{array}$ & $\mathrm{P}, \mathrm{S}, \mathrm{NS}$ & $\mathrm{O}, \mathrm{T}$ & Robayda 2906 \\
\hline E. citriodora Hook. & Eucalyptus & Tree, 1 & $\begin{array}{l}\text { RS, Wd } \\
(\mathrm{pl})\end{array}$ & All upazilas & $\mathrm{O}, \mathrm{T}$ & Robayda 1097 \\
\hline Psidium guajava $\mathrm{L}$. & Peara & Tree, $\mathrm{m}$ & ML & All upazilas & $\mathrm{Fr}$ & Robayda 1526 \\
\hline Syzygium cumini (L.) Skeels & Kalo Jam & Tree, 1 & FL, RS & All upazilas & Fr & Robayda 111 \\
\hline S. fruticosum Roxb. ex DC. & Banjam & Tree, $\mathrm{s}$ & SJ & S, NS, M, B & Fw, Fd & Robayda 362 \\
\hline S. jambos (L.) Alston & Golab-Jam & Tree, $\mathrm{s}$ & SJ, FL & All upazilas & Fw & Robayda 1368 \\
\hline $\begin{array}{l}\text { S. samarangense (Blume) Merr. \& L.M. } \\
\text { Perry }\end{array}$ & Jamrul & Tree, $\mathrm{s}$ & & All upazilas & $\mathrm{Fr}$ & Robayda 1487 \\
\hline \multicolumn{7}{|l|}{ PUNICACEAE Bercht. \& J. Presl } \\
\hline \multicolumn{7}{|l|}{ ONAGRACEAE Juss. } \\
\hline Ludwigia adscendens (L.) H. Hara & Keshordam & Herb, aq & WL & All upazilas & M & Robayda 1234 \\
\hline L. hyssopifolia (G. Don) Exell & Jhilmarich & Herb, er & $\mathrm{WL}, \mathrm{AF}$ & All upazilas & - & Robayda 139 \\
\hline
\end{tabular}




\begin{tabular}{|c|c|c|c|c|c|c|}
\hline Scientific name & Bangla name & Habit & Habitat & Distribution & Use & RSE \\
\hline $\begin{array}{l}\text { MELASTOMATACEAE Juss. } \\
\text { Melastoma malabathricum } \mathrm{L} \text {. } \\
\text { COMBRETACEAE } \mathrm{R} \text {. Br. }\end{array}$ & Ban tejpata & Shrub & RS, SJ & NS, S, B & M & Robayda 123 \\
\hline Combretum indicum (L.) DeFilipps & Madhurilata & Shrub, cl & SJ & $\mathrm{M}, \mathrm{B}, \mathrm{NS}, \mathrm{P}$ & $\mathrm{O}$ & Robayda 2448 \\
\hline $\begin{array}{l}\text { Terminalia arjuna (Roxb. ex DC.) Wight } \\
\& \text { Arn. }\end{array}$ & Arjun & Tree, 1 & RS & All upazilas & $\mathrm{M}, \mathrm{T}$ & Robayda 2738 \\
\hline T. catappa $\mathrm{L}$. & Kathbadam & Tree, $\mathrm{m}$ & RS & All upazilas & $\mathrm{M}, \mathrm{T}$ & Robayda 1109 \\
\hline $\begin{array}{l}\text { T. chebula Retz. } \\
\text { CORNACEAE Bercht. ex J. Presl }\end{array}$ & Haritoki & Tree, $\mathrm{m}$ & SJ & $\mathrm{B}, \mathrm{M}$ & M & Robayda 2832 \\
\hline $\begin{array}{l}\text { Alangium salviifolium (L. f.) Wangerin } \\
\text { RHIZOPHORACEAE Pers. }\end{array}$ & Akarkanta, Aikha & Tree, $\mathrm{s}$ & RS & $\mathrm{B}, \mathrm{NS}, \mathrm{P}, \mathrm{M}$ & M & Robayda 660 \\
\hline $\begin{array}{l}\text { Carallia brachiata (Lour.) Merr. Tree } \\
\text { OLACACEAE Juss. ex R. Br. }\end{array}$ & Roscow & Tree, $\mathrm{m}$ & RS, SJ & B, NS, M, S & $\mathrm{T}, \mathrm{M}$ & Robayda 2163 \\
\hline $\begin{array}{l}\text { Olax acuminata Wall. ex Benth. } \\
\text { LORANTHACEAE Juss. }\end{array}$ & Capsule gach & Shrub & SJ & $\mathrm{S}$ & M & Robayda 56 \\
\hline Dendrophthoe falcata Ettingsh. & Bajrangi & Shrub & T. T., SP & $\mathrm{P}, \mathrm{S}, \mathrm{M}, \mathrm{R}$ & - & Robayda 2693 \\
\hline D. pentandra (L.) Miq. & - & Shrub & T. T., SP & $\mathrm{P}$ & - & Robayda 2969 \\
\hline $\begin{array}{l}\text { Viscum monoicum Roxb. ex DC. } \\
\text { EUPHORBIACEAE Juss. }\end{array}$ & Bhanda & Shrub, ps & T. T., SP & NS, P & - & Robayda 2712 \\
\hline Acalypha indica $\mathrm{L}$. & Muktajhuri & Herb, er & RS, FL & $\mathrm{B}, \mathrm{P}, \mathrm{NS}, \mathrm{M}$ & $\mathrm{M}$ & Robayda 1935 \\
\hline $\begin{array}{l}\text { Chrozophora rottleri (Geiseler) A. Juss. } \\
\text { ex Spreng. }\end{array}$ & Khudi Okra & Herb, er & RS & NS, S, R & - & Robayda 550 \\
\hline Cnesmone javanica Blume & Pahari Bichhuti & Shrub & SJ & $\mathrm{P}, \mathrm{NS}$ & - & Robayda 51 \\
\hline $\begin{array}{l}\text { Codiaeum variegatum (L.) Rumph. ex A. } \\
\text { Juss. }\end{array}$ & Patabahar & Shrub & $\mathrm{FL}(\mathrm{pl})$ & All upazilas & $\mathrm{O}$ & Robayda 1497 \\
\hline Croton bonplandianus Baill. & Mircha & Herb, er & RS, FL & All upazilas & M & Robayda 1399 \\
\hline C. caudatus Geiseler & Sabarjala & Shrub & SJ & $\mathrm{S}, \mathrm{B}, \mathrm{M}, \mathrm{R}$ & M & Robayda 68 \\
\hline Euphorbia hirta L. & Dudhia & Herb, er & RS & All upazilas & M & Robayda 2073 \\
\hline E. neriifolia $\mathrm{L}$. & Mansasij & Shrub & SJ & $\mathrm{B}, \mathrm{S}, \mathrm{M}, \mathrm{P}$ & M & Robayda 1442 \\
\hline E. thymifolia $\mathrm{L}$. & Dudhiya & Herb, pr & RS & All upazilas & - & Robayda 2009 \\
\hline Jatropha curcas L. & Baghverenda & Shrub & RS, FL & B & M & Robayda 2609 \\
\hline J. gossypifolia $\mathrm{L}$. & Lalbherenda & Shrub & RS & $\mathrm{P}, \mathrm{NS}, \mathrm{B}$ & $\mathrm{M}$ & Robayda 848 \\
\hline Macaranga peltata (Roxb.) Müll. Arg. & Ratabura & Tree, $\mathrm{s}$ & $\mathrm{RS}, \mathrm{SJ}$ & All upazilas & - & Robayda 1700 \\
\hline Mallotus philippensis (Lam.) Müll. Arg. & Kamalaguli & Shrub & $\mathrm{RS}, \mathrm{SJ}$ & $\mathrm{B}, \mathrm{S}, \mathrm{M}$ & M & Robayda 2198 \\
\hline M. repandus (Rottler) Müll. Arg. & Gunti & Tree, $\mathrm{s}$ & SJ & $\mathrm{B}, \mathrm{S}, \mathrm{M}$ & M & Robayda 2382 \\
\hline Pedilanthus tithymaloides (L.) Poit. & Berachita & Shrub & FL & $\mathrm{B}, \mathrm{NS}, \mathrm{R}, \mathrm{P}$ & $\mathrm{O}$ & Robayda 2843 \\
\hline Ricinus communis $\mathrm{L}$. & Verenda & Shrub & RS & All upazilas & M & Robayda 1996 \\
\hline Suregada multiflora (A. Juss.) Baill. & Ban-narenga & Tree, $s$ & $\mathrm{RS}, \mathrm{SJ}$ & $\mathrm{B}, \mathrm{S}, \mathrm{NS}, \mathrm{M}$ & $\mathrm{T}$ & Robayda 1686 \\
\hline Trewia nudiflora $\mathrm{L}$. & Meragota & Tree, $\mathrm{m}$ & ML & All upazilas & M & Robayda 1990 \\
\hline PHYLLANTHACEAE Martinov & & & & & & \\
\hline Aporosa dioica (Roxb.) Müll. Arg. & Pat khorolla & Tree, $\mathrm{s}$ & SJ & $\mathrm{S}, \mathrm{M}, \mathrm{P}, \mathrm{NS}$ & - & Robayda 115 \\
\hline Baccaurea ramiflora Lour. & Latkan & Tree, $\mathrm{m}$ & FL & $\mathrm{B}, \mathrm{S}$ & Fr & Robayda 2145 \\
\hline $\begin{array}{l}\text { Breynia vitis-idaea (Burm. f.) C.E.C. } \\
\text { Fisch. }\end{array}$ & Lal Sitka & Shrub & SJ & All upazilas & - & Robayda 2319 \\
\hline Bridelia tomentosa Blume & Khoi & Shrub & SJ & S, B & - & Robayda 137 \\
\hline Flueggea virosa (Roxb. ex Willd.) Royle & Shikori & Shrub & $\mathrm{RS}, \mathrm{SJ}$ & All upazilas & M & Robayda 2356 \\
\hline Glochidion lanceolarium (Roxb.) Voigt & Anguti & Tree, $\mathrm{s}$ & $\mathrm{RS}, \mathrm{SJ}$ & All upazilas & - & Robayda 2538 \\
\hline Phyllanthus acidus (L.) Skeels & Orboroi & Shrub & RS & $\mathrm{NS}, \mathrm{B}, \mathrm{M}$ & $\mathrm{Fr}$ & Robayda 3107 \\
\hline P. emblica L. & Amloki & Tree, $\mathrm{m}$ & RS & All upazilas & Fr & Robayda 604 \\
\hline
\end{tabular}




\begin{tabular}{|c|c|c|c|c|c|c|}
\hline Scientific name & Bangla name & Habit & Habitat & Distribution & Use & RSE \\
\hline P. niruri $\mathrm{L}$. & Bhui amla & Herb, er & $\mathrm{RS}$ & All upazilas & $\mathrm{M}$ & Robayda 154 \\
\hline P. reticulatus Poir. & Chhitki & Shrub & FL, RS & All upazilas & M & Robayda 2004 \\
\hline P. urinaria $\mathrm{L}$. & Hajarmoni & Herb, er & FL, RS & All upazilas & M & Robayda 2391 \\
\hline \multicolumn{7}{|l|}{ RHAMNACEAE Juss. } \\
\hline Gouania leptostachya DC. & - & Shrub, cl & SJ & B & - & Robayda 2292 \\
\hline Ziziphus mauritiana Lam. & Boroi & Tree, $\mathrm{m}$ & RS, FL & All upazilas & $\mathrm{Fr}$ & Robayda 1528 \\
\hline Z. oenopolia (L.) Mill. & Bon boroi & Shrub & SJ & $\mathrm{B}, \mathrm{S}, \mathrm{M}$ & $\mathrm{Fd}$ & Robayda 118 \\
\hline \multicolumn{7}{|l|}{ VITACEAE Juss. } \\
\hline Ampelocissus barbata (Wall.) Planch. & Jarila Lahari & Shrub, cl & SJ & $\mathrm{B}, \mathrm{S}, \mathrm{M}, \mathrm{NS}$ & - & Robayda 2330 \\
\hline A. latifolia (Roxb.) Planch. & Gowalia lata & Herb, cl & SJ & $\mathrm{B}, \mathrm{S}, \mathrm{M}$ & - & Robayda 2211 \\
\hline Cissus adnata Roxb. & Alianga-lata & Shrub, cl & SJ & $\mathrm{B}, \mathrm{NS}$ & - & Robayda 1326 \\
\hline C. quadrangularis $\mathrm{L}$. & Harjora Lata & Herb, cl & $\mathrm{RS}$ & NS, M & M & Robayda 2923 \\
\hline Leea alata Edgew. & - & Shrub & SJ & $\mathrm{B}, \mathrm{S}$ & - & Robayda 2260 \\
\hline L. asiatica (L.) Ridsdale & Banchalita & Shrub & SJ & $\mathrm{S}, \mathrm{M}$ & - & Robayda 1241 \\
\hline L. indica (Burm. f.) Merr. & Kukur jihwa & Shrub & SJ & $\mathrm{S}$ & M & Robayda 2261 \\
\hline $\begin{array}{l}\text { Tetrastigma angustifolium (Roxb.) } \\
\text { Planch. }\end{array}$ & Nekung riubi & Herb, cl & $\mathrm{RS}$ & $\mathrm{R}$ & - & Robayda 3133 \\
\hline T. leucostaphylum (Dennst.) Alston & Horina -lata & Shrub, cl & $\mathrm{RS}$ & All upazilas & - & Robayda 2660 \\
\hline Vitis vinifera $\mathrm{L}$. & Angur & Shrub, cl & ML & NS, S, B, M & $\mathrm{Fr}$ & Robayda 2520 \\
\hline \multicolumn{7}{|l|}{ MALPIGHIACEAE Juss. } \\
\hline Hiptage benghalensis (L.) Kurz & Madhabilata & Shrub, cl & SJ & $\mathrm{S}$ & M & Robayda 110 \\
\hline \multicolumn{7}{|l|}{ SAPINDACEAE Juss. } \\
\hline Allophylus cobbe (L.) Forsyth f. & Rakhalchita & Shrub & SJ & $\mathrm{B}, \mathrm{S}$ & Fw & Robayda 2277 \\
\hline Dimocarpus longan Lour. & Kathlichu, Ashfal & Tree, $\mathrm{m}$ & SJ & $\mathrm{B}, \mathrm{S}, \mathrm{M}$ & - & Robayda 2545 \\
\hline Lepisanthes rubiginosa (Roxb.) Leenh. & Baraharina & Tree, $\mathrm{s}$ & $\mathrm{SJ}, \mathrm{FL}$ & $\begin{array}{l}\mathrm{B}, \mathrm{P}, \mathrm{NS}, \mathrm{S} \\
\mathrm{M}\end{array}$ & Fw & Robayda 2087 \\
\hline L. senegalensis (Juss. ex Poir.) Leenh. & Amjam & Shrub, & SJ & $\mathrm{S}, \mathrm{M}, \mathrm{P}$ & Fw & Robayda 2279 \\
\hline Litchi chinensis Sonn. & Lichu & Tree, 1 & ML & All upazilas & $\mathrm{Fr}$ & Robayda 1425 \\
\hline \multicolumn{7}{|l|}{ BURSERACEAE Kunth } \\
\hline $\begin{array}{l}\text { Protium serratum (Wall. ex Colebr.) } \\
\text { Engl. }\end{array}$ & Chitrica & Tree, $\mathrm{m}$ & SJ & $\mathrm{S}$ & $\mathrm{T}$ & Robayda 3053 \\
\hline \multicolumn{7}{|l|}{ ANACARDIACEAE R. Br. } \\
\hline Lannea coromandelica (Houtt.) Merr. & Jiga, Kafila & Tree, $\mathrm{m}$ & RS, ML & All upazilas & $\mathrm{T}, \mathrm{Fd}$ & Robayda 2135 \\
\hline Mangifera indica $\mathrm{L}$. & Aam & Tree, 1 & FL & All upazilas & $\mathrm{Fr}, \mathrm{T}$ & Robayda 1444 \\
\hline Spondias pinnata (L. f.) Kurz & Amra & Tree, 1 & $\mathrm{RS}$ & $\mathrm{S}, \mathrm{M}$ & $\mathrm{Fr}$ & Robayda 2907 \\
\hline S. purpurea L. & Beelati Amra & Tree, $\mathrm{m}$ & FL (pl) & All upazilas & $\mathrm{Fr}, \mathrm{M}$ & Robayda 752 \\
\hline \multicolumn{7}{|l|}{ MELIACEAE Juss. } \\
\hline $\begin{array}{l}\text { Aphanamixis polystachya (Wall.) R. } \\
\text { Parker }\end{array}$ & Pitraj, Roina & Tree, $\mathrm{m}$ & RS, SJ & All upazilas & $\mathrm{T}, \mathrm{M}$ & Robayda 2242 \\
\hline Azadirachta indica A. Juss. & Nim & Tree, 1 & RS, FL & All upazilas & $\mathrm{T}, \mathrm{M}$ & Robayda 1450 \\
\hline Melia azedarach $\mathrm{L}$. & Goranim & Tree, $\mathrm{m}$ & $\mathrm{RS}$ & All upazilas & $\mathrm{T}$ & Robayda 1957 \\
\hline Swietenia macrophylla King & Bara Mehagoni & Tree, 1 & Wd, RS & All upazilas & $\mathrm{T}$ & Robayda 2283 \\
\hline S. mahagoni (L.) Jacq. & Mehogoni & Tree, 1 & Wd, RS & All upazilas & $\mathrm{T}$ & Robayda 381 \\
\hline Toona ciliata M. Roem. & Toon & Tree, $\mathrm{m}$ & SJ & $\mathrm{S}, \mathrm{B}, \mathrm{M}$ & $\mathrm{T}$ & Robayda 22 \\
\hline \multicolumn{7}{|l|}{ RUTACEAE Juss. } \\
\hline Aegle marmelos (L.) Corrêa & Bel & Tree, $\mathrm{m}$ & FL & All upazilas & $\mathrm{Fr}, \mathrm{M}$ & Robayda 894 \\
\hline Citrus limon (L.) Osbeck & Gora lebu & Shrub & $\mathrm{RS}$ & All upazilas & $\mathrm{Fr}$ & Robayda 342 \\
\hline C. maxima (Burm.) Merr. & Jambura & Tree, 1 & FL & All upazilas & $\mathrm{Fr}$ & Robayda 1925 \\
\hline C. medica $\mathrm{L}$. & Lebu & Shrub & FL & All upazilas & $\mathrm{Fr}$ & Robayda 1560 \\
\hline
\end{tabular}




\begin{tabular}{|c|c|c|c|c|c|c|}
\hline Scientific name & Bangla name & Habit & Habitat & Distribution & Use & RSE \\
\hline Glycosmis pentaphylla (Retz.) DC. & Motkila & Shrub & RS, FL & All upazilas & $\mathrm{M}$ & Robayda 1982 \\
\hline Limonia acidissima $\mathrm{L}$. & Kothbel & Tree, 1 & RS & NS, S, B & $\mathrm{Fr}$ & Robayda 2722 \\
\hline Micromelum minutum Wight \& Arn. & Koroiphula & Tree, $\mathrm{s}$ & SJ & $\mathrm{B}, \mathrm{S}, \mathrm{M}$ & - & Robayda 2259 \\
\hline Murraya koenigii (L.) Spreng. & Currypata & Tree, $s$ & $\mathrm{RS}, \mathrm{SJ}$ & $\mathrm{B}, \mathrm{S}, \mathrm{M}$ & M & Robayda 1298 \\
\hline M. paniculata (L.) Jack & Kamini & Tree, $\mathrm{s}$ & RS & All upazilas & $\mathrm{O}, \mathrm{M}$ & Robayda 780 \\
\hline Zanthoxylum rhetsa DC & Bajna & Tree, $\mathrm{m}$ & SJ & All upazilas & $\mathrm{Ol}, \mathrm{T}$ & Robayda 2400 \\
\hline \multicolumn{7}{|l|}{ OXALIDACEAE R. Br. } \\
\hline Averrhoa bilimbi $\mathrm{L}$. & Bilimbi & Tree, $\mathrm{s}$ & FL & $\mathrm{NS}, \mathrm{B}, \mathrm{M}$ & $\mathrm{Fr}$ & Robayda 2089 \\
\hline A. carambola $\mathrm{L}$. & Kamranga & Tree, $\mathrm{m}$ & RS & All upazilas & Fr, M & Robayda 632 \\
\hline Oxalis corniculata $\mathrm{L}$. & Amrul & Herb, pr & FL, AF & All upazilas & $\mathrm{M}, \mathrm{V}$ & Robayda 1823 \\
\hline \multicolumn{7}{|l|}{ BALSAMINACEAE A. Rich. } \\
\hline Impatiens balsamina $\mathrm{L}$. & Dopati & Herb, er & RS & $\mathrm{P}, \mathrm{B}, \mathrm{M}$ & $\mathrm{O}$ & Robayda 2756 \\
\hline \multicolumn{7}{|l|}{ APIACEAE Lindl. } \\
\hline Centella asiatica (L.) Urb. & Thankuni & Herb, pr & FL, RS & All upazilas & M, V & Robayda 1279 \\
\hline Coriandrum sativum $\mathrm{L}$. & Dhonia & Herb, er & ML (cu) & $\mathrm{B}, \mathrm{S}, \mathrm{NS}, \mathrm{R}$ & $\mathrm{Sp}, \mathrm{M}$ & Robayda 1207 \\
\hline Daucus carota $\mathrm{L}$. & Gazor & Herb, er & ML (cu) & $\mathrm{P}, \mathrm{NS}, \mathrm{S}$ & $\mathrm{V}$ & Robayda 322 \\
\hline Eryngium foetidum $\mathrm{L}$. & Beelati Dhonia & Herb, er & FL & All upazilas & $\mathrm{Sp}$ & Robayda 1522 \\
\hline Hydrocotyle sibthorpioides Lam. & - & Herb, cr & FL & M & - & Robayda 1699 \\
\hline \multicolumn{7}{|l|}{ APOCYNACEAE Juss. } \\
\hline Allamanda cathartica $\mathrm{L}$. & Alakananda & Shrub & $\mathrm{RS}(\mathrm{pl})$ & $\mathrm{NS}, \mathrm{S}$ & $\mathrm{O}$ & Robayda 2764 \\
\hline Alstonia scholaris (L.) R. Br. & Chhatim & Tree, $\mathrm{m}$ & SJ, FL & All upazilas & M & Robayda 1972 \\
\hline Catharanthus roseus (L.) G. Don & Nayantara & Herb,er & RS & All upazilas & $\mathrm{O}, \mathrm{M}$ & Robayda 605 \\
\hline Carissa carandas $\mathrm{L}$. & Karamcha & Shrub & $\mathrm{FL}(\mathrm{pl})$ & $\mathrm{B}, \mathrm{S}, \mathrm{NS}, \mathrm{M}$ & Fr, M & Robayda 1418 \\
\hline $\begin{array}{l}\text { Holarrhena antidysenterica (L.) Wall. ex } \\
\text { A. DC. }\end{array}$ & Kurchi & Shrub & SJ & S & M & Robayda 387 \\
\hline Ichnocarpus frutescens (L.) W.T. Aiton & Shyamalata & Shrub, cl & SJ & NS, S, M, B & M & Robayda 2357 \\
\hline Nerium oleander L. & Raktakarabi & Shrub & RS & NS, S & $\mathrm{O}$ & Robayda 2788 \\
\hline Plumeria alba $\mathrm{L}$. & Gulachin & Tree, $\mathrm{s}$ & RS & NS, S, B & $\mathrm{O}$ & Robayda 3128 \\
\hline $\begin{array}{l}\text { Rauvolfia serpentina (L.) Benth. ex } \\
\text { Kurz }\end{array}$ & Sarpagandha & Herb, er & SJ & B & M & Robayda 2921 \\
\hline $\begin{array}{l}\text { Tabernaemontana divaricata (L.) R. Br. } \\
\text { ex Roem. \& Schult. }\end{array}$ & Tagar & Shrub & $\mathrm{RS}, \mathrm{SJ}$ & $\mathrm{B}, \mathrm{S}, \mathrm{M}, \mathrm{NS}$ & $\mathrm{O}, \mathrm{M}$ & Robayda 01 \\
\hline \multicolumn{7}{|l|}{ ASCLEPIADACEAE Borkh. } \\
\hline Calotropis gigantea $(\mathrm{L}$.) W.T. Aiton & Akand & Shrub & $\mathrm{RS}, \mathrm{SJ}$ & NS & M & Robayda 3061 \\
\hline C. procera (Aiton) W.T. Aiton & Akand & Shrub & $\mathrm{RS}, \mathrm{SJ}$ & NS, S, B, M & M & Robayda 1547 \\
\hline $\begin{array}{l}\text { Hemidesmus indicus (L.) R. Br. ex } \\
\text { Schult. }\end{array}$ & Anantamul & Shrub ,tw & FL & $\mathrm{P}, \mathrm{NS}$ & M & Robayda 1510 \\
\hline Marsdenia tenacissima (Roxb.) Moon & Jitti, Chitti & Shrub ,tw & ML, SJ & $\mathrm{B}, \mathrm{S}, \mathrm{M}$ & M & Robayda 2651 \\
\hline Telosma cordata (Burm. f.) Merr. & Kanja Lata & Shrub ,tw & SJ & NS & $\mathrm{Fb}$ & Robayda 2813 \\
\hline Dregea volubilis (L.f.) Benth. ex Hook.f. & Madhumaloti & Shrub, tw & $\mathrm{RS}, \mathrm{SJ}$ & NS, R & - & Robayda 3108 \\
\hline \multicolumn{7}{|l|}{ SOLANACEAE Juss. } \\
\hline Capsicum frutescens $\mathrm{L}$. & Lanka Morich & Herb, er & ML (cu) & All upazilas & $\mathrm{V}, \mathrm{Sp}$ & Robayda 232 \\
\hline Cestrum nocturnum $\mathrm{L}$. & Hasnahena & Shrub & RS & All upazilas & $\mathrm{O}$ & Robayda 2518 \\
\hline Datura metel L. & Dhutura & Herb, er & FL, RS & All upazilas & M & Robayda 850 \\
\hline Lycopersicon esculentum Mill. & Tomato & Herb, er & ML (cu) & All upazilas & $\mathrm{V}$ & Robayda 321 \\
\hline Nicotiana plumbaginifolia Viv. & Bon Tamak & Herb, er & FL & $\mathrm{P}, \mathrm{NS}, \mathrm{B}$ & - & Robayda 1594 \\
\hline Physalis angulata $\mathrm{L}$. & Fotka & Herb, er & FL & $\mathrm{P}, \mathrm{S}, \mathrm{NS}$ & M & Robayda 2719 \\
\hline P. minima $\mathrm{L}$. & Fotka & Herb, er & FL & All upazilas & M & Robayda 1635 \\
\hline Solanum americanum Mill. & Tit Begun & Herb, er & FL & $\mathrm{P}, \mathrm{S}$ & - & Robayda 2962 \\
\hline
\end{tabular}




\begin{tabular}{|c|c|c|c|c|c|c|}
\hline Scientific name & Bangla name & Habit & Habitat & Distribution & Use & RSE \\
\hline S. erianthum D. Don & - & Shrub & SJ & $\mathrm{B}, \mathrm{S}, \mathrm{M}$ & - & Robayda 2359 \\
\hline S. melongena $\mathrm{L}$. & Begun & Herb, er & FL (cu) & All upazilas & $\mathrm{V}$ & Robayda 1600 \\
\hline S. nigrum $\mathrm{L}$. & Tit Begun & Herb, er & FL & $\mathrm{B}, \mathrm{S}, \mathrm{M}$ & - & Robayda 1340 \\
\hline S. sisymbriifolium Lam. & Kanta begun & Herb, er & RS & All upazilas & - & Robayda 1794 \\
\hline S. torvum Sw. & Gota Begun & Shrub & RS, FL & $\mathrm{B}, \mathrm{S}, \mathrm{M}$ & - & Robayda 769 \\
\hline S. tuberosum L. & Aaloo & Herb, er & ML (cu) & All upazilas & $\mathrm{V}$ & Robayda 1507 \\
\hline \multicolumn{7}{|l|}{ CONVOLVULACEAE Juss. } \\
\hline Argyreia capitiformis (Poir.) Ooststr. & - & Shrub & SJ, RS & $\mathrm{P}$ & - & Robayda 2976 \\
\hline A. nervosa (Burm. f.) Bojer & Bara Dudhi & Shrub, cl & SJ, RS & $\mathrm{P}, \mathrm{B}, \mathrm{S}, \mathrm{M}$ & - & Robayda 2624 \\
\hline A. roxburghii (Wall.) Arn. ex Choisy & - & Shrub & SJ & NS & - & Robayda 2885 \\
\hline Cuscuta reflexa Roxb. & Sharnalata & Herb, ps & RS & All upazilas & - & Robayda 485 \\
\hline Evolvulus nummularius (L.) L. & Bhui Okra & Herb & RS, FL & All upazilas & - & Robayda 243 \\
\hline Ipomoea aquatica Forssk. & Kalmi Shak & Herb & WL & All upazilas & $\mathrm{V}$ & Robayda 1204 \\
\hline I. batatas (L.) Lam. & Misti Aloo & Herb & ML (cu) & All upazilas & $\mathrm{V}$ & Robayda 1486 \\
\hline I. fistulosa Mart. ex Choisy & Dhol kalmi & Shrub & ML, RS & All upazilas & - & Robayda 1989 \\
\hline $\begin{array}{l}\text { Jacquemontia paniculata (Burm. f.) } \\
\text { Hallier f. }\end{array}$ & - & Herb, tw & SJ & $\mathrm{P}, \mathrm{NS}$ & - & Robayda 809 \\
\hline $\begin{array}{l}\text { Merremia hederacea (Burm. f.) Hallier } \\
\text { f. }\end{array}$ & Kaladana & Herb, cl & RS, SJ & $\mathrm{P}$ & - & Robayda 838 \\
\hline \multicolumn{7}{|l|}{ MENYANTHACEAE Dumort. } \\
\hline Nymphoides hydrophylla (Lour.) Kuntze & - & Herb, aq & WL & $\mathrm{P}, \mathrm{S}, \mathrm{M}$ & - & Robayda 2731 \\
\hline N. indica (L.) Kuntze & Chandmala & Herb, aq & WL & $\mathrm{B}, \mathrm{S}, \mathrm{R}$ & - & Robayda 3059 \\
\hline \multicolumn{7}{|l|}{ HYDROPHYLLACEAE R. Br. } \\
\hline Hydrolea zeylanica (L.) Vahl & - & Herb, pr & WL & $\mathrm{S}, \mathrm{M}$ & - & Robayda 164 \\
\hline \multicolumn{7}{|l|}{ BORAGINACEAE Juss. } \\
\hline Cordia dichotoma G. Forst. & Bohal, Boula & Tree, 1 & SJ & $\mathrm{S}, \mathrm{NS}$ & $\mathrm{T}, \mathrm{M}$ & Robayda 527 \\
\hline C. serrata Juss. ex Lam. & - & Tree, $\mathrm{s}$ & SJ & $\mathrm{B}, \mathrm{M}, \mathrm{S}$ & Fw & Robayda 1318 \\
\hline Ehretia acuminata $\mathrm{R} . \mathrm{Br}$. & $\begin{array}{l}\text { Kala-aja, Kala- } \\
\text { huja }\end{array}$ & Tree, $\mathrm{s}$ & RS, SJ & All upazilas & M & Robayda 2435 \\
\hline \multicolumn{7}{|l|}{ VERBENACEAE J.St.-Hil. } \\
\hline Duranta erecta $\mathrm{L}$. & Kanta mehendi & Shrub & RS & All upazilas & $\mathrm{O}$ & Robayda 925 \\
\hline Lantana camara L. & $\begin{array}{l}\text { Lantana, Kutus } \\
\text { Kanta }\end{array}$ & Shrub & RS, FL & All upazilas & $\mathrm{O}$ & Robayda 1585 \\
\hline $\begin{array}{l}\text { Lippia alba (Mill.) N.E. Br. ex Britton \& } \\
\text { P. Wilson }\end{array}$ & Pichas ban & Shrub & RS, ML & All upazilas & - & Robayda 2071 \\
\hline Phyla nodiflora (L.) Greene & Bhuiokra & Herb, dc & ML & $\mathrm{P}, \mathrm{B}, \mathrm{NS}, \mathrm{R}$ & M & Robayda 1856 \\
\hline \multicolumn{7}{|l|}{ LAMIACEAE Martinov } \\
\hline Anisomeles indica (L.) Kuntze & Gobura & Herb, er & RS, FL & $\mathrm{B}, \mathrm{S}, \mathrm{M}$ & M & Robayda 2470 \\
\hline Callicarpa macrophylla Vahl & Bormala & Shrub & SJ & B & $\mathrm{M}$ & Robayda 2219 \\
\hline Clerodendrum chinense (Osbeck) Mabb. & Hazari beli & Shrub & SJ & $\mathrm{B}, \mathrm{S}, \mathrm{M}$ & $\mathrm{O}$ & Robayda 2365 \\
\hline C. indicum (L.) Kuntze & Bamunhatti & Shrub & SJ, ML & $\mathrm{B}, \mathrm{S}, \mathrm{M}$ & $\mathrm{M}, \mathrm{O}$ & Robayda 2218 \\
\hline C. infortunatum $\mathrm{L}$. & Bhat,Ghetu & Shrub & RS, ML & All upazilas & M & Robayda 1973 \\
\hline Coleus scutellarioides (L.) Benth. & Patabahar & Herb, er & $\mathrm{ML}(\mathrm{pl})$ & All upazilas & $\mathrm{O}$ & Robayda 744 \\
\hline Gmelina arborea Roxb. & Gamari & Tree, 1 & SJ, FL & $\mathrm{B}, \mathrm{S}, \mathrm{M}$ & $\mathrm{T}$ & Robayda 2740 \\
\hline Hyptis suaveolens (L.) Poit. & Tokma & Herb, er & RS, FL & All upazilas & M & Robayda 1855 \\
\hline Leonurus sibiricus $\mathrm{L}$. & Roktodron & Herb, er & RS & All upazilas & M & Robayda 2364 \\
\hline Leucas aspera (Willd.) Link & Shetadron & Herb, er & $\begin{array}{l}\text { AF, RS, } \\
\text { FL }\end{array}$ & All upazilas & M & Robayda 1810 \\
\hline
\end{tabular}




\begin{tabular}{|c|c|c|c|c|c|c|}
\hline Scientific name & Bangla name & Habit & Habitat & Distribution & Use & RSE \\
\hline $\begin{array}{l}\text { L. indica (L.) R. Br. ex Sm. } \\
\text { Sm }\end{array}$ & Shetadron & Herb, er & $\begin{array}{l}\text { AF, RS, } \\
\text { FL }\end{array}$ & $\mathrm{B}, \mathrm{R}$ & $\mathrm{M}$ & Robayda 3065 \\
\hline Mentha arvensis $\mathrm{L}$. & Pudina & Herb, pr & FL (cu) & $\mathrm{P}, \mathrm{NS}$ & $\mathrm{V}, \mathrm{M}$ & Robayda 344 \\
\hline Ocimum americanum $\mathrm{L}$. & Ban Tulsi & Herb, er & FL & $\mathrm{R}, \mathrm{M}, \mathrm{B}$ & M & Robayda 1006 \\
\hline O. tenuiflorum $\mathrm{L}$. & Tulsi & Herb, er & FL & All upazilas & M & Robayda 2575 \\
\hline Pogostemon auricularius (L.) Hassk. & - & Herb, er & RS & S & - & Robayda 2537 \\
\hline P. quadrifolius (Benth.) F. Muell. & - & Herb, er & ML & M & - & Robayda 916 \\
\hline P. stellatus (Lour.) Kuntze & - & Herb, er & WL & $\mathrm{R}$ & - & Robayda 3131 \\
\hline Premna esculenta Roxb. & Lalana & Shrub & SJ & B & - & Robayda 2231 \\
\hline Tectona grandis L. f. & Segun & Tree, 1 & $\mathrm{RS}, \mathrm{Wd}$ & $\mathrm{B}, \mathrm{S}, \mathrm{P}, \mathrm{R}$ & $\mathrm{T}$ & Robayda 642 \\
\hline $\begin{array}{l}\text { Volkameria inermis L. } \\
\text { OLEACEAE Hoffeanns. \& Link }\end{array}$ & Banjui & Shrub & RS, ML & M & $\mathrm{O}, \mathrm{M}$ & Robayda 2711 \\
\hline $\begin{array}{l}\text { Jasminum multiflorum (Burm. f.) } \\
\text { Andrews }\end{array}$ & Chameli & Shrub & $\mathrm{RS}(\mathrm{pl})$ & NS, S & $\mathrm{O}$ & Robayda 2762 \\
\hline J. sambac (L.) Aiton & Beli & Shrub & SJ & $\mathrm{B}, \mathrm{S}, \mathrm{M}$ & $\mathrm{O}$ & Robayda 1084 \\
\hline $\begin{array}{l}\text { Nyctanthes arbor-tristis L. } \\
\text { PLANTAGINACEAE Juss. }\end{array}$ & Sheuli & Tree, $\mathrm{s}$ & FL, SJ & All upazilas & $\mathrm{O}$ & Robayda 735 \\
\hline Limnophila aromatica (Lam.) Merr. & Pani karpur & Herb, er & $\mathrm{AF}$ & $\mathrm{S}, \mathrm{R}, \mathrm{M}, \mathrm{B}$ & - & Robayda 1165 \\
\hline L. repens (Benth.) Benth. & - & Herb, er & $\mathrm{AF}$ & S & - & Robayda 131 \\
\hline L. sessiliflora Blume & - & Herb, er & $\mathrm{AF}$ & $\mathrm{S}, \mathrm{B}, \mathrm{M}$ & - & Robayda 130 \\
\hline Mecardonia procumbens (Mill.) Small & - & Herb, er & $\mathrm{FL}, \mathrm{AF}$ & $\mathrm{P}, \mathrm{B}, \mathrm{M}$ & - & Robayda 832 \\
\hline Scoparia dulcis $\mathrm{L}$. & Bandhane & Herb, er & FL, RS & All upazilas & M & Robayda 2345 \\
\hline $\begin{array}{l}\text { LINDERNIACEAE Borsch, Kai Müll. } \\
\text { \& Eb. Fisch. }\end{array}$ & & & & & & \\
\hline Lindernia anagallis (Burm.f.) Pennell & - & Herb, pr & $\mathrm{AF}, \mathrm{ML}$ & $\mathrm{B}, \mathrm{S}, \mathrm{M}, \mathrm{P}$ & - & Robayda 1630 \\
\hline L. antipoda (L.) Alston & - & Herb, pr & $\mathrm{RS}, \mathrm{AF}$ & All upazilas & - & Robayda 168 \\
\hline L. crustacea (L.) F. Muell. & - & Herb, pr & RS, FL & All upazilas & M & Robayda 2485 \\
\hline L. rotundifolia (L.) Alston & - & Herb, pr & $\mathrm{AF}, \mathrm{ML}$ & All upazilas & - & Robayda 2465 \\
\hline L. ruellioides (Colsm.) Pennell & - & Herb, pr & ML & M & - & Robayda 2533 \\
\hline L. viscosa (Hornem.) Merr. & - & Herb, er & RS & $\mathrm{P}$ & - & Robayda 3011 \\
\hline MAZACEAE Reveal & & & & & & \\
\hline $\begin{array}{l}\text { Mazus pumilus (Burm. f.) Steenis } \\
\text { ACANTHACEAE Juss. }\end{array}$ & - & Herb, er & RS & $\mathrm{P}, \mathrm{R}, \mathrm{S}$ & - & Robayda 1427 \\
\hline Dipteracanthus prostratus (Poir.) Nees & - & Herb, er & RS & $\mathrm{B}, \mathrm{NS}, \mathrm{P}, \mathrm{M}$ & M & Robayda 1683 \\
\hline Ecbolium ligustrinum (Vahl) Vollesen & Udujati & Shrub & SJ & B & M & Robayda 2246 \\
\hline Hemigraphis hirta T. Anderson & Buripan & Herb, er & RS, FL & $\mathrm{P}, \mathrm{B}, \mathrm{M}$ & - & Robayda 290 \\
\hline Hygrophila phlomoides Nees & - & Herb, er & $\mathrm{AF}$ & $\mathrm{P}$ & M & Robayda 1578 \\
\hline H. polysperma (Roxb.) T. Anderson & - & Herb, er & $\mathrm{WL}, \mathrm{AF}$ & $\mathrm{S}, \mathrm{B}, \mathrm{M}$ & - & Robayda 1166 \\
\hline Justicia adhatoda $\mathrm{L}$. & Basak & Shrub & SJ, RS & $\mathrm{B}, \mathrm{S}, \mathrm{M}, \mathrm{NS}$ & M & Robayda 643 \\
\hline J. diffusa Willd. & - & Herb, pr & RS & $\mathrm{R}$ & - & Robayda 3097 \\
\hline J. gendarussa Burm. f. & Jagatmadan & Shrub & FL & All upazilas & M & Robayda 87 \\
\hline $\begin{array}{l}\text { Lepidagathis inaequalis C.B. Clarke ex } \\
\text { Elmer }\end{array}$ & - & Herb, pr & RS & NS & - & Robayda 2857 \\
\hline Nelsonia canescens (Lam.) Spreng. & Paramul & Herb, pr & RS & All upazilas & - & Robayda 42 \\
\hline Phaulopsis imbricata (Forssk.) Sweet & - & Herb, pr & RS & All upazilas & - & Robayda 1369 \\
\hline Phlogacanthus curviflorus (Wall.) Nees & - & Shrub & SJ & $\mathrm{B}, \mathrm{M}$ & $\mathrm{O}$ & Robayda 2129 \\
\hline Ruellia tuberosa $\mathrm{L}$. & Chotpoty & Herb, er & RS & $\mathrm{B}, \mathrm{M}$ & M & Robayda 2791 \\
\hline Rungia pectinata (L.) Nees & Pindi & Herb, er & RS & All upazilas & M & Robayda 88 \\
\hline Staurogyne argentea Wall. & - & Herb, er & FL & NS & - & Robayda 2932 \\
\hline
\end{tabular}




\begin{tabular}{|c|c|c|c|c|c|c|}
\hline Scientific name & Bangla name & Habit & Habitat & Distribution & Use & RSE \\
\hline Thunbergia erecta (Benth.) T. Anderson & Nil Ghanta & Shrub & RS & $\mathrm{B}$ & $\mathrm{O}$ & Robayda 2414 \\
\hline T. grandiflora Roxb. & Nil lata & Herb, cl & SJ & $\mathrm{S}$ & - & Robayda 91 \\
\hline \multicolumn{7}{|l|}{ PEDALIACEAE R. Br. } \\
\hline $\begin{array}{l}\text { Sesamum indicum } \mathrm{L} \text {. } \\
\text { BIGNONIACEAE Juss. }\end{array}$ & Til & Herb, er & RS & NS, R, B, S & $\mathrm{Ol}$ & Robayda 2086 \\
\hline Oroxylum indicum (L.) Kurz & Kanaidingi, Sona & Tree, $\mathrm{m}$ & SJ & M, NS, S & M & Robayda 2224 \\
\hline \multicolumn{7}{|l|}{ LENTIBULARIACEAE Rich. } \\
\hline $\begin{array}{l}\text { Utricularia foliosa } \mathrm{L} \text {. } \\
\text { CAMPALUNACEAE Juss. }\end{array}$ & & Robayda 2732 \\
\hline Lobelia radicans Thunb. & - & Herb, er & FL & $\mathrm{P}, \mathrm{B}, \mathrm{R}, \mathrm{M}$ & M & Robayda 2258 \\
\hline L.terminalis C.B. Clarke & - & Herb, pr & $\mathrm{AF}$ & M & - & Robayda 1173 \\
\hline \multicolumn{7}{|l|}{ RUBIACEAE Juss. } \\
\hline Borreria ocymoides (Burm. f.) DC. & - & Herb, er & ML & $\mathrm{P}, \mathrm{B}$ & - & Robayda 3009 \\
\hline Catunaregam spinosa (Thunb.) Tirveng. & Monkata & Shrub & SJ & $\mathrm{S}, \mathrm{M}$ & - & Robayda 2902 \\
\hline Chassalia curviflora (Wall.) Thwaites & Hel Gass & Shrub & SJ & B & - & Robayda 2212 \\
\hline Coffea benghalensis B. Heyne ex Schult. & Bancofee & Shrub & SJ & All upazilas & - & Robayda 2151 \\
\hline Dentella serpyllifolia Wall. ex Craib & Bhuipat & Herb, pr & FL & All upazilas & - & Robayda 2214 \\
\hline Gardenia jasminoides $\mathrm{J}$. Ellis & Gandharaj & Shrub & SJ, RS & All upazilas & $\mathrm{O}$ & Robayda 464 \\
\hline Geophila repens (L.) I.M. Johnst. & Koodi Munkooni & Herb, pr & SJ & $\mathrm{B}, \mathrm{M}$ & M & Robayda 2118 \\
\hline Hedyotis corymbosa (L.) Lam. & Khet papra & Herb, pr & FL & All upazilas & M & Robayda 1945 \\
\hline H. diffusa Willd. & - & Herb, pr & RS, ML & All upazilas & - & Robayda 2639 \\
\hline Hymenodictyon orixense (Roxb.) Mabb. & Bhutum & Tree, $\mathrm{m}$ & RS & M & $\mathrm{T}$ & Robayda 2622 \\
\hline Ixora acuminata Roxb. & Rangan & Shrub & SJ & $\mathrm{S}, \mathrm{M}$ & - & \\
\hline I. chinensis Lam. & Rangan & Shrub & $\mathrm{RS}(\mathrm{pl})$ & All upazilas & $\mathrm{O}$ & Robayda 627 \\
\hline I. cuneifolia Roxb. & Rangan & Shrub & SJ & $\mathrm{B}, \mathrm{S}, \mathrm{M}$ & - & Robayda 1722 \\
\hline Meyna spinosa Roxb. ex Link & Monkata & Shrub & SJ & $\mathrm{S}, \mathrm{NS}, \mathrm{R}$ & - & Robayda 61 \\
\hline Morinda angustifolia Roxb. & $\begin{array}{l}\text { Daru haridra, } \\
\text { Banomali }\end{array}$ & Shrub & SJ & $\mathrm{B}, \mathrm{S}, \mathrm{M}$ & M & Robayda 06 \\
\hline $\begin{array}{l}\text { Mussaenda erythrophylla Schumach. \& } \\
\text { Thonn. }\end{array}$ & Machhenda & Shrub & $\mathrm{RS}(\mathrm{pl})$ & NS, S & $\mathrm{O}$ & Robayda 2767 \\
\hline Neolamarckia cadamba (Roxb.) Bosser & Kadam & Tree, $\mathrm{m}$ & RS & All upazilas & $\mathrm{O}$ & Robayda 1359 \\
\hline Psychotria monticola Kurz & - & Shrub & SJ & B & - & Robayda 2326 \\
\hline Richardia scabra L. & - & Herb, pr & FL, RS & $\mathrm{P}, \mathrm{R}$ & - & Robayda 2215 \\
\hline Spermacoce articularis L. f. & Ahtharogia & Herb, er & FL, RS & All upazilas & - & Robayda 773 \\
\hline S. latifolia Aubl. & Ghuiojhil shak & Herb, er & FL, RS & All upazilas & - & Robayda 2236 \\
\hline \multicolumn{7}{|l|}{ ASTERACEAE Bercht. \& J. Presl } \\
\hline Acmella radicans (Jacq.) R.K. Jansen & - & Herb, er & RS & $S$ & - & Robayda 1670 \\
\hline Ageratum conyzoides $\mathrm{L}$. & Fulkuri & Herb, er & RS & All upazilas & - & Robayda 1976 \\
\hline Blumea densiflora DC. & Kukshim & Herb, er & RS, FL & $\mathrm{P}, \mathrm{S}, \mathrm{NS}$ & - & Robayda 263 \\
\hline B. membranacea DC. & Kukshim & Herb, er & RS, FL & All upazilas & - & Robayda 1808 \\
\hline Centipeda minima (L.) A. Braun \& Asch. & Nakchikni & Herb, pr & WL, ML & $\mathrm{S}, \mathrm{NS}, \mathrm{P}$ & - & Robayda 1672 \\
\hline $\begin{array}{l}\text { Chromolaena odorata (L.) R.M. King \& } \\
\text { H. Rob. }\end{array}$ & Asamlata & Shrub & RS, SJ & All upazilas & M & Robayda 1928 \\
\hline Cosmos sulphureus Cav. & Cosmos & Herb, er & RS & All upazilas & $\mathrm{O}$ & Robayda 2759 \\
\hline $\begin{array}{l}\text { Cotula hemisphaerica (Roxb.) Wall. ex } \\
\text { Benth. }\end{array}$ & Babuni & Herb, pr & ML & S, NS, B, M & - & Robayda 1161 \\
\hline $\begin{array}{l}\text { Crassocephalum crepidioides (Benth.) S. } \\
\text { Moore }\end{array}$ & - & Herb, er & $\mathrm{AF}, \mathrm{FL}$ & $\mathrm{P}$ & - & Robayda 2235 \\
\hline Eclipta alba (L.) Hassk. & Kalo keshi & Herb, pr & FL, RS & All upazilas & M & Robayda 2306 \\
\hline
\end{tabular}




\begin{tabular}{|c|c|c|c|c|c|c|}
\hline Scientific name & Bangla name & Habit & Habitat & Distribution & Use & RSE \\
\hline Elephantopus scaber $\mathrm{L}$. & Hastipadi & Herb, er & FL & $\mathrm{M}, \mathrm{S}$ & $\mathrm{M}$ & Robayda 54 \\
\hline Emilia sonchifolia (L.) DC. & Mechitra & Herb, er & RS & NS, P & - & Robayda 2460 \\
\hline Enydra fluctuans DC. & Helencha & Herb, aq & WL & All upazilas & $\mathrm{V}$ & Robayda 1216 \\
\hline Gnaphalium luteoalbum $\mathrm{L}$. & Bara Kamra & Herb, er & $\mathrm{ML}, \mathrm{AF}$ & All upazilas & - & Robayda 194 \\
\hline Grangea maderaspatana (L.) Poir. & Nemuti & Herb, pr & ML & $\mathrm{NS}, \mathrm{P}, \mathrm{B}, \mathrm{S}$ & M & Robayda 169 \\
\hline Mikania cordata (Burm. f.) B.L. Rob. & Taralata & Herb, cl & $\mathrm{RS}, \mathrm{SJ}$ & All upazilas & M & Robayda 1986 \\
\hline Parthenium hysterophorus L. & - & Herb, er & RS & NS & - & Robayda 2967 \\
\hline $\begin{array}{l}\text { Pseudelephantopus spicatus (B. Juss. ex } \\
\text { Aubl.) C.F. Baker }\end{array}$ & - & Herb, er & RS, FL & $\begin{array}{l}\mathrm{P}, \mathrm{NS}, \mathrm{B}, \mathrm{S}, \\
\mathrm{M}\end{array}$ & - & Robayda 1791 \\
\hline Sonchus wightianus DC. & - & Herb, er & FL & $\mathrm{P}$ & - & Robayda 1760 \\
\hline Spilanthes calva DC. & Surja kannya & Herb, dc & FL, RS & All upazilas & M & Robayda 2177 \\
\hline Synedrella nodiflora (L.) Gaertn. & Nakphul & Herb, er & FL, RS & All upazilas & M & Robayda 1824 \\
\hline Tridax procumbens $\mathrm{L}$. & Tridhara & Herb, pc & RS & All upazilas & M & Robayda 1919 \\
\hline Vernonia cinerea (L.) Less. & Shialmutra & Herb, er & RS, FL & All upazilas & - & Robayda 1389 \\
\hline Wedelia trilobata (L.) Hitchc. & Baharibhringaraj & Herb, pc & RS, FL & $\mathrm{B}, \mathrm{NS}$ & $\mathrm{O}$ & Robayda 2455 \\
\hline Xanthium indicum J. Koenig ex Roxb. & Ghagra & Herb, er & ML & $\mathrm{P}, \mathrm{B}, \mathrm{M}, \mathrm{S}$ & M & Robayda 933 \\
\hline
\end{tabular}

LEGEND: Habit. $\mathrm{aq}=$ aquatic, $\mathrm{cl}=$ climbing, $\mathrm{dc}=$ decumbent, $\mathrm{er}=$ erect, $\mathrm{l}=$ large, $\mathrm{m}=$ medium, $\mathrm{pc}=$ procumbent, $\mathrm{pr}=$ prostrate, $\mathrm{ps}=$ parasitic, $\mathrm{s}=$ small, $\mathrm{sc}=$ scandent, $\mathrm{tw}=$ twiner. Habitat. $\mathrm{AF}=$ Agricultural Field, $\mathrm{cu}=\mathrm{Cultivated}, \mathrm{FL}=$ Fallow Land, $\mathrm{ML}=$ Marginal Land, $\mathrm{Pl}=$ Planted, $\mathrm{RS}=$ Roadsides, $\mathrm{SJ}=$ Scrub Jungle, $\mathrm{SP}=$ Semi Parasitic, $\mathrm{TT}=$ Tree Trunk, WL = Wet Land, WD = Wood Land. Distrib. = Distribution. $\mathrm{B}=$ Belabo, $\mathrm{M}=$ Monohordi, NS = Narsingdi Sadar, $\mathrm{P}$ $=$ Palash, $\mathrm{R}=$ Raipura, $\mathrm{S}=$ Shibpur. Use $\mathrm{B} \& \mathrm{Th}=$ Binding $\&$ Thatching Material, $\mathrm{Dy}=$ dye, $\mathrm{Fb}=$ Fiber, $\mathrm{Fd}=$ fodder, $\mathrm{Fr}=$ Fruit, $\mathrm{Fw}=$ Fuel Wood, $\mathrm{M}=$ Medicine, $\mathrm{O}=$ Ornamental, $\mathrm{Ol}=\mathrm{Oil}, \mathrm{Pu}=$ pulse, $\mathrm{Sp}=$ Spice, $\mathrm{T}=$ Timber, and $\mathrm{V}=$ Vegetable. RSE $=$ Representative Specimens Examined

\section{Results and Discussion}

This study has confirmed the occurrence of total 468 species of dicotyledons (Magnoliopsida) under 326 genera and 85 families in Narsingdi district. Among these families, 30 were represented by single species each and only 17 by more than $10(10-53)$ species. Fabaceae with 53 species of 37 genera was recognized as the largest family in this study area, followed by Asteraceae with 25 species under 24 genera and Rubiaceae with 21 species of 17 genera. Ficus L. with eight species was the largest genus in this area, which was followed by Persicaria (L.) Mill. and Solanum L. with seven species each and Lindernia All. with six species, Phyllanthus L. with five species and Acacia Mill., Amaranthus L., Senna Mill., Sida L. and Trichosanthes L. with four species each. Rest of the families were consisted of two or three species each. These data on the genera and species indicate that Narsingdi district is still rich in dicotyledons. The study area was dominated by the herbs, comprised of $230(49.14 \%)$ species that were followed by the trees of 120 (25.64\%) species, and the shrubs of $118(25.21 \%)$ species. In this area, the roadsides, harbouring the highest number of species (226 species), were the most common type of habitat and the agricultural fields with relatively lower number of species (19 species) were the occasional habitat.

In Narsingdi district, total 200 species were commonly distributed in its all upazilas, 58 species in two upazilas, 83 species in three upazilas, 58 species in four upazilas and only four species in five upazilas. 14 species exclusively occured in Palash, 13 in each of Belabo and Shibpur, 10 in Narsingdi Sadar, nine in Monohordi and only six in Raipura upazila. Belabo upazila accommodated total 351 species, which was followed by Shibpur, Monohordi, Narsingdi Sadar, Palash and Raipura upazilas harbouring 344, 317, 313, 291 and 248 species, respectively. Based on these data, the dicotyledonous flora was considered relatively richer in Belabo, Shibpur, Monohordi and Narsingdi Sadar upazilas, in comparison to those of Palash and Raipura upazilas. However, if these species enumeration are considered in term of total land areas of these upazilas, 
then their sequence turns in to Belabo, followed by Palash, Monohordi, Shibpur, Narsingdi Sadar, and Raipura. According to Jaccard coefficient, the similarity in six upazilas of Narsingdi district in harboring the dicotyledonous species was $75.47 \%$ (Fig. 1). It indicates that the species composition in these upazilas are more similar rather than different if they are compared all together. However, the similarities in between the two upazilas only varied from 9.03\% (in Monohordi and Raipura upazilas) to 50\% (in Monohordi and Belabo upazilas).

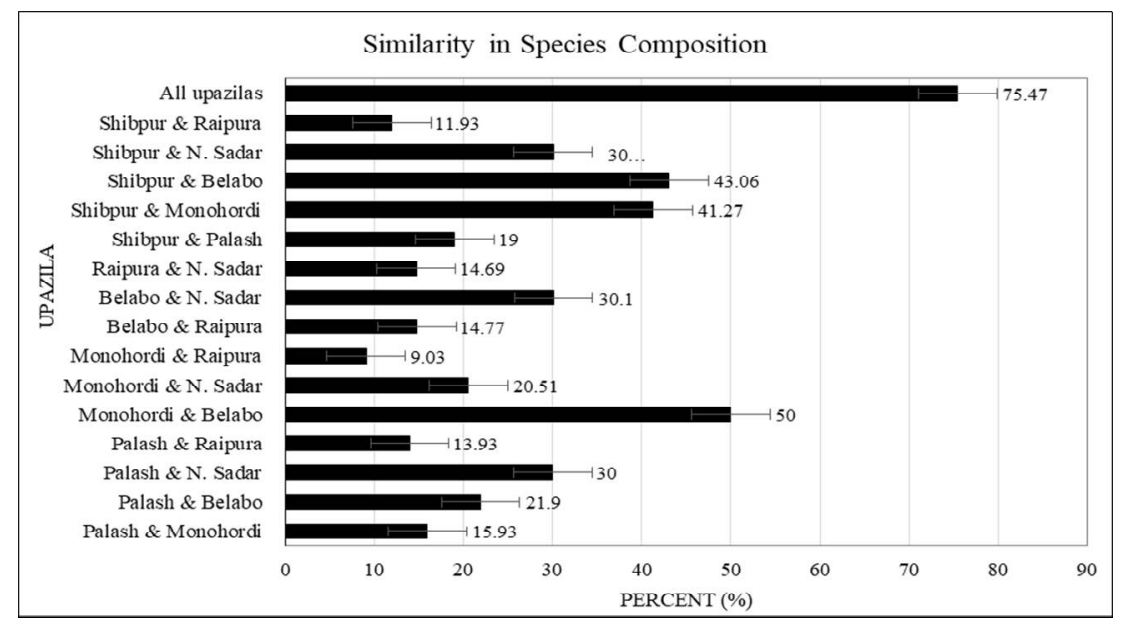

Fig. 1. Similarity in species composition in the upazilas of Narsingdi district based on Jaccard coefficient (Jaccard, 1912).

These rercords on dicotyledonous species of six upazilas of Narsindi district are higher than the enumeration of this plant group in some upazilas of other districts by the previous studies, (Islam et al., 2009; Rahman et al., 2012; Sarker et al., 2013; Sajib et al., 2014; Mahmudah et al., 2017; Rahman et al., 2019). Whereas, the record on dicot species of Raipura upazila by this study is somewhat lower than that of Munshiganj Sadar upazila by Rahman et al., 2013, though those of other upazilas of Narsindi district are notably higher. Considering the size of the study area, the dicotyledonous flora of whole Narsingdi district seems richer in comparison to those of Sundarbans, Patuakhali district and Rajshahi district, as reported by Rahman et al. (2017), Sultana (2012) and Rahman (2013), respectively. Whereas, the dicotyledones of this district is relatively poorer than those of few forest areas viz., Sitapahar Reserve Forest (Uddin et al., 1998; Rashid and Chowdhury, 2013), Satchari National Park (Arefin et al., 2011); Hazarikhil Wildlife Sanctuary (Rahman, 2017), and Rajkandi Reserve Forest (Haque et al., 2018). These variation in species composition of different areas might be due to different natural, biogeographical and anthropogenic factors, drivers or threats etc. functioning in these area, and the methods and intenseness of the studies as well.

During this study, total 333 dicot species of Narsingdi district were figured out as economically useful. The major categories of these species were medicinal (167 species), timber (46 species), fruit (37 species), ornamental (60 species), vegetable (43 species), fodder (9 species), fiber (11 species), fuel wood (12 species), oil yielding (five species), pulse (four species) and spices (three species). Among these species, 67 were recognized to be useful in two to three categories. The total number of dicot species (468) recorded from Narsingdi district during this study was $17.84 \%$ of the total 2623 species, and the number of dicot families (89) was $56.32 \%$ of 
the total 158 families reported for Bangladesh by Ahmed et al. (2008-2009). However, these proportions of the dicots of this district would be decreased if the flora of Bangladesh is explored completely.

One threatened species Rauvolfia serpentina (L.) Benth. ex Kurz found in the study area is already included in Red Data Book of Vascular Plants of Bangladesh (Khan et al., 2001). The species Geophila repens (L.) I.M. Johnst., rediscovered (Khanam et al., 2018) from this area, wasis found as threatened there, which is not yet included in the Red Data Book of Vascular Plants of Bangladesh. In the study area, the populations of Geophila repens (L.) I. M. Johnst. and Rauvolfia serpentina (L.) Benth. ex Kurz were comprised of total 50 and nine individuals, respectively. G. repens and R. serpentina were confined to two and four localities with the Extent of Occurrence (EOO) and Area of Occupancy (AOO) of $250 \mathrm{~km}^{2}$ and $25 \mathrm{~km}^{2}$, and $280 \mathrm{~km}^{2}$ and 50 $\mathrm{km}^{2}$, respectively. Another species Acmella radicans (Jacq.) R.K. Jansen was found in five localities of Shibpur upazila with total 40 individuals only. Its EOO and AOO were estimated as $120 \mathrm{~km}^{2}$ and $15 \mathrm{~km}^{2}$, respectively. The regeneration in these three species was poor and their localities were under the regular stress of habitat destruction due to which these species have been considered as Endangered (E) in Narsingdi district. Both of Acmella radicans and Geophila repens might be included in the Red Data Book of Vascular Plants of Bangladesh and IUCN Red List for Bangladesh following exploration in other areas of this country. Different anthropogenic activities continuously performed by increased human population, especially vegetation clearing, unnecessary firing, unplanned agricultural extension, over exploitation of natural resources, and habitat fragmentation and depletion; soil erosion; invasion of some exotic species (e.g. Acacia auriculiformis, Chromolaena odorata, Eucalyptus camaldulensis, Mikania cordata and Parthenium hysterophorus); poor regeneration in some species; lack of awareness in the local people; and lack of proper management programs etc. are the major functional threats to the flora of the study area.

The taxonomic information provided by this study on the species of dicotyledons extant in Narsingdi district can serve as an important guiding database to track the trend of changes in the floristic composition, plant species diversity and vegetation in course of time due to natural and anthropogenic stresses, contribute in undertaking appropriate biodiversity conservation initiatives and plant resourse-based socioeconomic development and help in estimating the impacts of climate change in this area. This study highly recommends for regular inventory, monitoring and specific research programs on the flora and plant diversity of this district and implementation of appropriate conservation measures for the threatened and depleting plant species of this area.

\section{Acknowledgements}

The authors gratefully acknowledge the Bangladesh University Grants Commissions for awarding the $\mathrm{Ph} \mathrm{D}$ Fellowship to the first author for conducting her research including this study. They are grateful to the authority of Bangladesh National Herbarium (DACB) for allowing access to their libraries and relevant herbarium materials. The authors are also thankful to the Reviewers of the Journal for their critical review of the manuscript.

\section{References}

Ahmed, Z.U., Begum, Z.N.T., Hassan, M.A., Khondker, M., Kabir, S.M.H., Ahmed, M., Ahmed, A.T.A., Rahman, A.K.T. and Haque, E.U. (Eds). 2008-2009. Encyclopedia of Flora and Fauna of Bangladesh, Vols. 6-10 and 12, Asiatic Society of Bangladesh, Dhaka.

Alam, M.S., Hassan, M.A. and Uddin, M.Z. 2006. A Preliminary Checklist of the Angiospermic flora of Ghagotia Union under Kapasia Upazila in Gazipur District, Bangladesh. Bangladesh J. Plant Taxon 13(2): 155-170. 
Ara, H., Khan, B. and Uddin, S.N. (Eds). 2013. Red Data Book of Vascular Plants of Bangladesh. Bangladesh National Herbarium, Dhaka, pp. 1-280.

Arefin, M.K., Rahman, M.M., Uddin, M.Z. and Hassan, M.A. 2011. Angiosperm flora of Satchari National Park, Habiganj, Bangladesh. Bangladesh J. Plant Taxon. 18(2): 117-140.

Bridson, D.M. and Forman, F. 1989. In: Bridson, D.M. and Forman, F. (Eds). The Herbarium Handbook. Royal Botanic Gardens, Kew, pp. 214.

Chowdhury, M.S.U. 1996. Bangladesh: country report to the FAO international technical conference on plant genetic resources, Leipzig, 1-112.

Cronquist, A. 1981. An integrated system of classification of flowering plants. Columbia University Press, New York, pp. 1-1262.

Ghani, A. 1998. Medicinal Plants of Bangladesh with Chemical Constituents and Uses. Asiatic Society of Bangladesh, pp. 1-467.

Haque, A.K.M.K., Khan, S.A., Uddin, S.N. and Rahim, M.A. 2016. Taxonomic Checklist of the Pteridophytes of Rajkandi Reserve Forest, Moulvibazar, Bangladesh. Jahangirnagar University J. Biol. Sci. 5(2): 27-40.

Haque, A.K.M.K., Khan, S.A., Uddin, S.N. and Shetu, S.S. 2018. An annotated checklist of the Angiospermic flora of Rajkandi Reserve Forest of Moulvibazar, Bangladesh. Bangladesh J. Plant Taxon. 25(2): 187-207.

Hooker, J.D. 1872-1897. The Flora of British India. Vols. 1-7. L. Reeve \& Co., Ashford, Kent, UK.

Huq, A.M. 1986. Plant Names of Bangladesh. Bangladesh National Herbarium, BARC, Dhaka, Bangladesh, pp. 1-289.

IPNI. 2017. International Plant Names Index. Published on the Internet http://www.ipni.org, The Royal Botanic Gardens, Kew, Harvard University Herbaria \& Libraries and Australian National Botanic Gardens. Accessed on 21 May 2017.

Islam, M.R., Uddin, M.Z. and Hassan, M.A. 2009. An Assessment of the Angiospermic Flora of Ramgarh Upazila of Khagrachhari District, Bangladesh. Bangladesh J. Plant Taxon. 16(2): 115-140.

IUCN Standards and Petitions Committee. 2019. Guidelines for Using the IUCN Red List Categories and Criteria. Version 14. Prepared by the Standards and Petitions Committee, https://www. iucnredlist.org/ resources/redlistguidelines, pp. 1-113.

Jaccard, P. 1912. The distribution of the flora of the alpine zone. New Phytologist 11: 37-50.

Kanjilal, U.N., Kanjilal, P.C., De, R.N. and Das, A. 1934. Flora of Assam. Vol. 1. Indian reprint 1982. A Von Book Company, Delhi, pp. 1-386.

Kanjilal, U.N., Kanjilal, P.C. and Das, A. 1938-1940. Flora of Assam. Vols. 2-4. Reprint 1982. A Von Book Company, Delhi.

Khan, M.S. 1977. Onagraceae. In: Khan, M.S. (ed.). Flora of Bangladesh. Fasc. 6: 1-10. Bangladesh National Herbarium, BARC, Dhaka.

Khan, M.S. and Huq, A.M. 2001. The vascular flora of Chunati wildlife sanctuary in south Chittagong. Bangladesh J. Plant Taxon. 8(1): 47-64.

Khan, M.S., Rahman, M.M. and Ali, M.A. (Eds). 2001. Red Data Book of Vascular Plants of Bangladesh, Vol. 1. Bangladesh National Herbarium, Dhaka, Bangladesh, pp. 1-179.

Khanam, R., Khan, S.A. and Rahim, A. 2018. Rediscovery of Geophila repens (L.) I. M. Johnst. (Rubiaceae) in Bangladesh. Jahangirnagar University J. Biol. Sci. 7(2): 121-125.

Mahmudah, Z., Islam, M.M, Haque, T. and Uddin, M.Z. 2017. Taxonomic Enumeration of Angiosperm Flora of Sreenagar Upazila, Munshigang, Dhaka, Bangladesh. J. Asiat. Soc. Bangladesh, Sci. 43(2): 161-172.

Nasir, E. and Ali, S.I. (Eds). 1980-2005. Flora of Pakistan. Vols. 1-209. University of Karachi, Karachi, Pakistan.

Pasha, M.K. and Uddin, S.B. 2013. Dictionary of Plant Names of Bangladesh (Vascular Plants). Janokalyan Prokashani, Chittagong, pp. 1-320.

Prain, D. 1903. Bengal Plants. Vols. 1 and 2. Reprint 1963. Botanical Survey of India, Calcutta. 
Rahman, A.H.M.M. 2013. Angiospermic Flora of Rajshahi District, Bangladesh. American Journal of Life Sciences 1(3): 105-112.

Rahman, M.A. 2017. Plant Diversity in Hazarikhil Wildlife Sanctuary of Chittagong and its Conservation Management. J. biodivers. Conserv. Bioresour. Manag. 3(2): 43-56.

Rahman, M.O., Begum, M. and Ullah, M.W. 2013. Angiosperm Flora of Sadar Upazila of Munshiganj District, Bangladesh. Bangladesh J. Plant Taxon. 20(2): 213-231.

Rahman, M.O. and Hassan, M.A. 1995. Angiospermic flora of Bhawal National Park, Gazipur (Bangladesh). Bangladesh J. Plant Taxon. 2(1\&2): 47-80.

Rahman, M.O., Antara, R.T., Begum, M. and Hassan, M.A. 2012. Floristic diversity of Dhamrai upazila of Dhaka with emphasis on medicinal plants. Bangladesh J. Bot. 41(1): 71-85.

Rahman, M.O., Sayma, N.J. and Begum, M. 2019. Angiospermic flora of Gafargaon upazila of Mymensingh district focusing on medicinally important species. Bangladesh J. Plant Taxon. 26(2): 269-283.

Rahman, M.S., Hossain, M.G., Khan, S.A. and Uddin, S.N. 2015. An Annotated Checklist of the Vascular Plants of Sundarban Mangrove forest of Bangladesh. Bangladesh J. Plant Taxon. 22(1): 17-41.

Rashid, M.H.U. and Chowdhury, M.A.I. 2013. Additions to the Angiosperm Flora in the Sitapahar Reserve Forest of Kaptai, Rangamati, Bangladesh. Bangladesh J. Plant Taxon. 20(2): 255-257.

Rashid, S.H. and Mia, M.M.K. 2001. Angiospermic flora of Madhupur National Park, Tangail, Bangladesh. Bangladesh J. Plant Taxon. 8(2): 63-82.

Rashid, S.H., Rahman, M.M. and Hossain, A.B.M.E. 1995. An inventory of the under growth resources in Chandra Sal forest at Gazipur, Bangladesh. Bangladesh J. Life Sci. 7(1\&2): 111-118.

Sajib, N.H., Uddin, S.B. and Islam, M.M. 2014. Angiospermic Plant Diversity of Subarnachar Upazila in Noakhali, Bangladesh. J. Asiat. Soc. Bangladesh, Sci. 40(1): :39-60.

Sarker, K., Islam, M.R., Uddin, M.Z., and Hassan, M.A. 2013. Angiosperm Flora of Manikgonj Sadar Upazila, Bangladesh. J. Asiat. Soc. Bangladesh, Sci. 39(2): 147-166.

Shetu, S.S., Khan, S.A. and Uddin, S.N. 2018. Checklist of Angiosperms extant in Mirpur area of Dhaka City, Jahangirnagar University J. Biol. Sci. 7(2): 47-64.

Singh, H.B. and Subramaniam, B. 2008. Field Manual on Herbarium Techniques. National Institute of Science Communication and Information Resources, pp. 1-297.

Sultana, M. 2012. Taxonomic and ethnobatanical studies on the angiospermic flora of Patuakhali district in Bangladesh. Ph.D. Thesis (Unpublished). Department of Botany, University of Dhaka.

The Plant List, 2013. The Plant List, a working list of all plant species. Version $1.1<$ http://www.t heplantlist. org/>. Accessed on 15 October 2018.

TROPICOS, 2017. Tropicos.org. <www.tropicos.org>. Missouri Botanical Garden, Saint Louis, Missouri, USA. Accessed on 14 July 2019.

Tutul, E., Uddin, M.Z., Rahman, M.O. and Hassan, M.A., 2010, Angiospermic flora of Runctia Sal forest, Bangladesh, II. Magnoliopsida (Dicots). Bangladesh J. Plant Taxon. 17(1): 33-53.

Uddin, S.N., Khan, M.S., Hassan, M.A. and Alam, M.K. 1998. An annotated checklist of angiospermic flora of Sitapahar at Kaptai in Bangladesh. Bangladesh J. Plant Taxon. 5(1): 13-46.

Van Valkenburg, J.L.C.H. and Bunyapraphatsara. N. (Eds). 2002. Plant Resources of South-East Asia, No. 12(2). Medicinal and Poisonous Plants 2. Prosea Foundation, Bogor, Indonesia, $782 \mathrm{pp}$.

Wu, Z.Y. and Raven, P.H. (Eds). 1994-2001. Flora of China, Vols. 8, 15-18 and 24. Missouri Botanical Garden Press, St. Louis, USA.

Wu, Z.Y., Raven, P.H. and Hong, D.Y. (Eds). 1999-2013. Flora of China, Vols. 2-7, 9-14, 19-23 and 25. Missouri Botanical Garden Press, St. Louis. 